\title{
Chronic myeloid leukemia model with periodic pulsed treatment
}

\author{
Helal Mohamed ${ }^{1}$, Abdelkader Lakmeche ${ }^{2}$ and Fethi Souna ${ }^{3}$ \\ Biomathematics Laboratory \\ Univ Sidi-Bel-Abbès \\ Sidi-Bel-Abbès \\ Algeria \\ ${ }^{1}$ mhelal_abbes@yahoo.fr, ${ }^{2}$ lakmeche@yahoo.fr and ${ }^{3}$ fethiou91@gmail.com
}

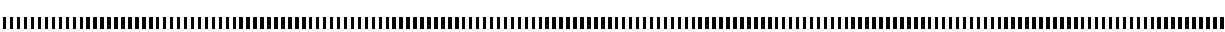

ABSTRACT. In this work we develop a mathematical model of chronic myeloid leukemia including treatment with instantaneous effects. Our analysis focuses on the values of growth rate $\gamma$ which give either stability or instability of the disease free equilibrium. If the growth rate $\gamma$ of sensitive leukemic stem cells is less than some threshold $\gamma^{*}$, we obtain the stability of disease free equilibrium which means that the disease is eradicated for any period of treatment $\tau_{0}$. Otherwise, for $\gamma$ great than $\gamma^{*}$, the period of treatment must be less than some specific value $\tau_{0}^{*}$. In the critical case when the period of treatment is equal to $\tau_{0}^{*}$, we observe a persistence of the tumor, which means that the disease is viable.

KEYWORDS : Positive solution, impulsive differential equation, Fixed point theorem, Bifurcation.

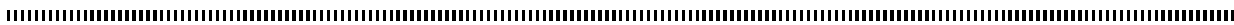


In this work we are interested by the study of a mathematical model of chronic myeloid leukemia. The chronic myeloid leukemia is a cancer of the bone marrow and blood which is characterized by an abnormal proliferation of blood cells, usually white blood cells. This disease is a myelo-proliferative disorder characterized by the expansion of a clone of hematopoietic cells that carries the Philadelphia chromosome $(\mathrm{Ph})$. The $\mathrm{Ph}$-chromosome results from a reciprocal translocation between the long arms of chromosomes 9 and 22 . In this paper, we study a mathematical model of chronic myeloid leukemia (CML) under treatment, the model studied here is inspired from [9] and [16].

Several recent works have been developed to study the dynamics of CML under chemotherapy treatment, see ([14], [15], [16] and [17]).

More specifically, we consider the following mathematical model which is an extension of the model proposed in [9]. In our model, we assume that normal (resp. leukemic) cells differentiate through two stages of their life cycle, beginning with normal (resp. sensitive leukemic) stem cells which produce normal (resp. leukemic) progenitor cells. The mathematical form of the system we shall investigate is the following

$$
\left\{\begin{array}{l}
\dot{x}_{0}=\left(\beta-a_{x}-\beta_{0} x_{0}-\lambda\left(x_{1}+y_{1}\right)\right) x_{0} \\
\dot{x}_{1}=a_{x} x_{0}-d_{1} x_{1} \\
\dot{y}_{0}=\left(\gamma-a_{y}-\gamma_{0} y_{0}-\lambda\left(x_{1}+\alpha y_{1}\right)\right) y_{0} \\
\dot{y}_{1}=a_{y} y_{0}-d_{2} y_{1}
\end{array}\right.
$$

with initial conditions

$$
x_{0}(0) \geq 0, x_{1}(0) \geq 0, y_{0}(0) \geq 0 \text { and } y_{1}(0) \geq 0 \text {. }
$$

Throughout this paper, we assume the following conditions

$$
a_{y}<\gamma
$$

and

$$
a_{x}<\beta
$$

The conditions (3) and (4) are necessary to obtain the biological meanings of the state variables $x_{0}, x_{1}, y_{0}$ and $y_{1}$.

In [9], well-posedness of (1), (2) is proved and stability (local and global) of equilibria is investigated. In fact, the disease free equilibrium $E_{f}=\left(x_{0}^{*}, \frac{a_{x}}{d_{1}} x_{0}^{*}, 0,0\right)$ is locally asymptotically stable for growth rate of sensitive leukemic stem cells $\gamma>\gamma^{*}:=a_{y}+$ $\lambda \frac{a_{x}}{d_{1}} x_{0}^{*}$ and unstable for $\gamma<\gamma^{*}$ where $x_{0}^{*}=\frac{\left(\beta-a_{x}\right) d_{1}}{\beta_{0} d_{1}+\lambda a_{x}}$.

In this paper, we consider the model above including chemotherapeutic treatment. We obtain the following model 


$$
\begin{aligned}
& \dot{x}_{0}(t)=F_{1}\left(x_{0}, x_{1}, y_{0}, y_{1}\right) \\
& \dot{x}_{1}(t)=F_{2}\left(x_{0}, x_{1}, y_{0}, y_{1}\right), \\
& \dot{y}_{0}(t)=F_{3}\left(x_{0}, x_{1}, y_{0}, y_{1}\right), \\
& \dot{y}_{1}(t)=F_{4}\left(x_{0}, x_{1}, y_{0}, y_{1}\right)
\end{aligned}
$$

for $t>0$ and $t \neq t_{i}$, where $t_{i}$ is the time of the $i^{t h}$ treatment,

$$
\begin{aligned}
& F_{1}\left(x_{0}, x_{1}, y_{0}, y_{1}\right)=\left(\beta-a_{x}-\beta_{0} x_{0}-\lambda\left(x_{1}+y_{1}\right)\right) x_{0}, \\
& F_{2}\left(x_{0}, x_{1}, y_{0}, y_{1}\right)=a_{x} x_{0}-d_{1} x_{1}, \\
& F_{3}\left(x_{0}, x_{1}, y_{0}, y_{1}\right)=\left(\gamma-a_{y}-\gamma_{0} y_{0}-\lambda\left(x_{1}+\alpha y_{1}\right)\right) y_{0}, \text { and } \\
& F_{4}\left(x_{0}, x_{1}, y_{0}, y_{1}\right)=a_{y} y_{0}-d_{2} y_{1} .
\end{aligned}
$$

For $t=t_{i}$ we have

$$
\begin{aligned}
& x_{0}\left(t_{i}^{+}\right)=\Theta_{1}\left(x_{0}\left(t_{i}\right), x_{1}\left(t_{i}\right), y_{0}\left(t_{i}\right), y_{1}\left(t_{i}\right)\right), \\
& x_{1}\left(t_{i}^{+}\right)=\Theta_{2}\left(x_{0}\left(t_{i}\right), x_{1}\left(t_{i}\right), y_{0}\left(t_{i}\right), y_{1}\left(t_{i}\right)\right), \\
& y_{0}\left(t_{i}^{+}\right)=\Theta_{3}\left(x_{0}\left(t_{i}\right), x_{1}\left(t_{i}\right), y_{0}\left(t_{i}\right), y_{1}\left(t_{i}\right)\right), \\
& y_{1}\left(t_{i}^{+}\right)=\Theta_{4}\left(x_{0}\left(t_{i}\right), x_{1}\left(t_{i}\right), y_{0}\left(t_{i}\right), y_{1}\left(t_{i}\right)\right),
\end{aligned}
$$

where $x_{j}\left(t_{i}^{+}\right)=\lim _{t \rightarrow t_{i}} x_{j}(t)$ and $y_{j}\left(t_{i}^{+}\right)=\lim _{t \rightarrow t_{i}} y_{j}(t),(j=0,1)$ are the

$$
\begin{array}{rl}
t>t_{i} & t>t_{i}
\end{array}
$$

size of $x_{j}$ just after the $i^{t h}$ treatment. In our case we have

$$
\begin{aligned}
& \Theta_{1}\left(x_{0}\left(t_{i}\right), x_{1}\left(t_{i}\right), y_{0}\left(t_{i}\right), y_{1}\left(t_{i}\right)\right)=x_{0}\left(t_{i}\right), \\
& \Theta_{2}\left(x_{0}\left(t_{i}\right), x_{1}\left(t_{i}\right), y_{0}\left(t_{i}\right), y_{1}\left(t_{i}\right)\right)=x_{1}\left(t_{i}\right), \\
& \Theta_{3}\left(x_{0}\left(t_{i}\right), x_{1}\left(t_{i}\right), y_{0}\left(t_{i}\right), y_{1}\left(t_{i}\right)\right)=T_{0} y_{0}\left(t_{i}\right), \\
& \Theta_{4}\left(x_{0}\left(t_{i}\right), x_{1}\left(t_{i}\right), y_{0}\left(t_{i}\right), y_{1}\left(t_{i}\right)\right)=T_{1} y_{1}\left(t_{i}\right) .
\end{aligned}
$$

The variables and parameters are

$x_{0}$ : biomass of the normal stem cells,

$x_{1}$ : biomass of normal progenitor cells,

$y_{0}$ : biomass of sensitive leukemic stem cells,

$y_{1}$ : biomass of sensitive leukemic progenitor cells,

$\beta_{0}:$ death rate of the normal stem cells,

$\gamma_{0}$ : death rate of sensitive leukemic stem cells,

$\beta$ : growth rate of normal stem cells,

$\gamma:$ growth rate of sensitive leukemic stem cells,

$\lambda:$ competitive parameter of the stem and progenitor cells,

$a_{x}:$ produce rate of the normal stem cells,

$a_{y}$ : produce rate of the sensitive leukemic stem cells,

$d_{1}$ : death rate of the normal progenitor cells,

$d_{2}$ : death rate of the sensitive leukemic progenitor cells,

$\alpha$ : competition parameter $(0<\alpha<1)$, 
$T_{0}(<1)$ : survival fraction of sensitive leukemic stem cells,

$T_{1}(<1)$ : survival fraction of sensitive leukemic progenitor cells, and

$\tau:$ is the time of the first treatment, it's the period between two successive injections, that is $t_{i}=i \tau, i \in \mathbf{N}$.

We obtain a special kind of differential equations called impulsive differential equations (see [1]-[6] and [8]).

Our main objective is to study the existence of steady states of (1) and their stability. Our paper is organized as follow; In the next section we give a mathematical analysis of our model, we study the well-posedness of (1), the existence of the steady states, and the stability of the trivial, chronic, blast and non pathological steady states. Further, we analyze the bifurcation of chronic periodic solutions of (1). Section two is devoted to numerical simulations, in the third section we give some conclusions. The last section is an appendix, where we give calculations needed for the previous sections.

\section{Mathematical analysis of the model}

\subsection{Well-posedness}

Theorem 1.1 The model (5)-(12) has a unique global positive solution for all positive initial conditions

PROOF. -

Since $F_{i},(i=1, \ldots, 4)$ are smooth, then from the Cauchy-Lipschitz theorem we have the local existence and uniqueness of the solutions of (5)-(8). Since the solutions are bounded then the solution is global in $\left[0, t_{1}\right]$.

The system (5)-(8) is quasi positive because for all $x_{0}, x_{1}, y_{0}$ and $y_{1} \in \mathbf{R}_{+}$we have $F_{1}\left(0, x_{1}, y_{0}, y_{1}\right)=0 \geq 0, F_{2}\left(x_{0}, 0, y_{0}, y_{1}\right)=a_{x} x_{0} \geq 0, F_{3}\left(x_{0}, x_{1}, 0, y_{1}\right)=0 \geq 0$ and $F_{4}\left(x_{0}, x_{1}, y_{0}, 0\right)=a_{y} y_{0} \geq 0$, so we have a unique positive global solution in $\left[0, t_{1}\right]$. By recurrence we can prove that $\forall k \in \mathbf{N}^{*}$, we have a unique positive global solution in the interval $\left[t_{k}, t_{k+1}\right]$. Hence, we have the existence of a unique positive global solution of (5)-(12).

\subsection{Stability of the disease free equilibrium $E_{f}$}

We can show that $\zeta(t):=\zeta_{0}=\left(\frac{\left(\beta-a_{x}\right) d_{1}}{\beta_{0} d_{1}+\lambda a_{x}}, \frac{\lambda a_{x}\left(\beta-a_{x}\right)}{\beta_{0} d_{1}+\lambda a_{x}}, 0,0\right)=E_{f}$ is a constant equilibrium of (5)-(12), it is called trivial solution.

To study the stability of $\zeta$ we use the same approach of fixed point process than in [7] and [11]-[13].

Since solutions of (5)-(8) exist globally in $\mathbf{R}_{+}$and are nonnegative (see [9]) we have

$$
X(t)=\Phi\left(t, X_{0}\right), t \geq 0
$$

where $X(t)=\left(x_{0}, x_{1}, y_{0}, y_{1}\right)(t), X(0)=X_{0}$ and $\Phi$ is the flow associated to (5)-(12).

The term $X\left(\tau^{+}\right)$denotes the state of the population after the treatment, $X\left(\tau^{+}\right)=\Theta(X(\tau))=$ $\Theta\left(\Phi\left(\tau, X_{0}\right)\right)$.

To have periodic solution we must have $X\left(\tau^{+}\right)=X_{0}$ that is $X_{0}=\Theta\left(\Phi\left(\tau, X_{0}\right)\right)$.

Let $\Psi$ be the operator defined by

$$
\Psi\left(\tau, X_{0}\right)=\Theta\left(\Phi\left(\tau, X_{0}\right)\right)
$$


and denote by $D_{X} \Psi$ the derivative of $\Psi$ with respect to $X$. Then $X=\Phi\left(., X_{0}\right)$ is a $\tau$-periodic solution of (5)-(12) if and only if

$$
\Psi\left(\tau, X_{0}\right)=X_{0}
$$

i.e. $X_{0}$ is a fixed point of $\Psi(\tau,$.$) , and it is exponentially stable if and only if the spectral$ radius $\rho\left(D_{X} \Psi(\tau,).\right)$ is strictly less than 1 (see [10]).

We need the following hypothesis

$(H 1): a_{x}<\beta<a_{x}+\frac{\left(\beta_{0} d_{1}+\lambda a_{x}\right)\left[\left(\beta_{0} d_{1}+2 \lambda a_{x}\right)-2 \sqrt{\lambda a_{x}\left(\beta_{0} d_{1}+\lambda a_{x}\right)}\right]}{\beta_{0}^{2} d_{1}}$ or
$\beta>a_{x}+\frac{\left(\beta_{0} d_{1}+\lambda a_{x}\right)\left[\left(\beta_{0} d_{1}+2 \lambda a_{x}\right)+2 \sqrt{\lambda a_{x}\left(\beta_{0} d_{1}+\lambda a_{x}\right)}\right]}{\beta_{0}^{2} d_{1}}$.

We deduce the following results.

\section{Theorem 1.2}

Let $(H 1)$ be satisfied.

1) If $\gamma \leq \gamma^{*}$, then the trivial solution $\zeta$ is exponentially stable for all $\tau_{0}>0$.

2) If $\gamma>\gamma^{*}$, then the trivial solution $\zeta$ is exponentially stable for $\tau_{0}<\tau_{0}^{*}:=$ $\frac{\ln T_{0}}{-\gamma+a_{y}+\lambda \frac{a_{x}}{d_{1}} x_{0}^{*}}$ and unstable for $\tau_{0}>\tau_{0}^{*}$.

PROOF. -

We have $D_{X} \Psi\left(\tau, X_{0}\right)=D_{X} \Theta\left(\Phi\left(\tau, X_{0}\right)\right) \frac{\partial \Phi}{\partial X}\left(\tau, X_{0}\right)$.

Then, for $X_{0}=\zeta_{0}$ and $\tau=\tau_{0}$ we have

$$
\begin{aligned}
D_{X} \Psi\left(\tau_{0}, \zeta_{0}\right) & =D_{X} \Theta\left(\Phi\left(\tau_{0}, \zeta_{0}\right)\right) \frac{\partial \Phi}{\partial X}\left(\tau_{0}, \zeta_{0}\right) \\
& =\left(\begin{array}{cccc}
1 & 0 & 0 & 0 \\
0 & 1 & 0 & 0 \\
0 & 0 & T_{0} & 0 \\
0 & 0 & 0 & T_{1}
\end{array}\right)\left(\begin{array}{cccc}
\frac{\partial \Phi_{1}\left(\tau_{0}, \zeta_{0}\right)}{\partial x_{0}} & \frac{\partial \Phi_{1}\left(\tau_{0}, \zeta_{0}\right)}{\partial x_{1}} & \frac{\partial \Phi_{1}\left(\tau_{0}, \zeta_{0}\right)}{\partial y_{0}} & \frac{\partial \Phi_{1}\left(\tau_{0}, \zeta_{0}\right)}{\partial y_{1}} \\
\frac{\partial \Phi_{2}\left(\tau_{0}, \zeta_{0}\right)}{\partial x_{0}} & \frac{\partial \Phi_{2}\left(\tau_{0}, \zeta_{0}\right)}{\partial x_{1}} & 0 & 0 \\
0 & 0 & \frac{\partial \Phi_{3}\left(\tau_{0}, \zeta_{0}\right)}{\partial y_{0}} & 0 \\
0 & 0 & \frac{\partial \Phi_{1}\left(\tau_{0}, \zeta_{0}\right)}{\partial y_{0}} & \frac{\partial \Phi_{1}\left(\tau_{0}, \zeta_{0}\right)}{\partial y_{1}}
\end{array}\right) \\
& =\left(\begin{array}{cccc}
\frac{\partial \Phi_{1}\left(\tau_{0}, \zeta_{0}\right)}{\partial x_{0}} & \frac{\partial \Phi_{1}\left(\tau_{0}, \zeta_{0}\right)}{\partial x_{1}} & \frac{\partial \Phi_{1}\left(\tau_{0}, \zeta_{0}\right)}{\partial y_{0}} & \frac{\partial \Phi_{1}\left(\tau_{0}, \zeta_{0}\right)}{\partial y_{1}} \\
\frac{\partial \Phi_{2}\left(\tau_{0}, \zeta_{0}\right)}{\partial x_{0}} & \frac{\partial \Phi_{2}\left(\tau_{0}, \zeta_{0}\right)}{\partial x_{1}} & 0 & 0 \\
0 & 0 & \frac{T_{0} \frac{\partial \Phi_{3}\left(\tau_{0}, \zeta_{0}\right)}{\partial y_{0}}}{0} & 0 \\
0 & 0 & \frac{\partial \Phi_{1}\left(\tau_{0}, \zeta_{0}\right)}{\partial y_{0}} & T_{1} \frac{\partial \Phi_{1}\left(\tau_{0}, \zeta_{0}\right)}{\partial y_{1}}
\end{array}\right) .
\end{aligned}
$$

The equilibrium $\zeta$ is exponentially stable if and only if the spectral radius is less than one. We have

$$
\operatorname{det}\left(D_{X} \Psi\left(\tau_{0}, \zeta_{0}\right)-\mu I\right)=\left(T_{0} \frac{\partial \Phi_{3}\left(\tau_{0}, \zeta_{0}\right)}{\partial y_{0}}-\mu\right)\left(T_{1} \frac{\partial \Phi_{4}\left(\tau_{0}, \zeta_{0}\right)}{\partial y_{1}}-\mu\right) \chi(\mu)
$$


where

$\chi(\mu)=\mu^{2}-\left(\frac{\partial \Phi_{1}}{\partial x_{0}}\left(\tau_{0}, \zeta_{0}\right)+\frac{\partial \Phi_{2}}{\partial x_{1}}\left(\tau_{0}, \zeta_{0}\right)\right) \mu+\left(\frac{\partial \Phi_{1}}{\partial x_{0}}\left(\tau_{0}, \zeta_{0}\right) \frac{\partial \Phi_{2}}{\partial x_{1}}\left(\tau_{0}, \zeta_{0}\right)-\frac{\partial \Phi_{1}}{\partial x_{1}}\left(\tau_{0}, \zeta_{0}\right) \frac{\partial \Phi_{2}}{\partial x_{0}}\left(\tau_{0}, \zeta_{0}\right)\right)$.

From (16) and (17), the equilibrium $\zeta=E_{f}$ is exponentially stable if and only if $T_{0}\left|\frac{\partial \Phi_{3}}{\partial y_{0}}\left(\tau_{0}, \zeta_{0}\right)\right|<1, T_{1}\left|\frac{\partial \Phi_{4}}{\partial y_{1}}\left(\tau_{0}, \zeta_{0}\right)\right|<1$ and $\left|\mu_{ \pm}\right|<1$. Where

$$
\mu_{ \pm}=\frac{\left(\frac{\partial \Phi_{1}}{\partial x_{0}}\left(\tau_{0}, \zeta_{0}\right)+\frac{\partial \Phi_{2}}{\partial x_{1}}\left(\tau_{0}, \zeta_{0}\right)\right) \pm \sqrt{\Delta}}{2}
$$

and

$$
\Delta=\left(\frac{\partial \Phi_{1}}{\partial x_{0}}\left(\tau_{0}, \zeta_{0}\right)-\frac{\partial \Phi_{2}}{\partial x_{1}}\left(\tau_{0}, \zeta_{0}\right)\right)^{2}+4 \frac{\partial \Phi_{1}}{\partial x_{1}}\left(\tau_{0}, \zeta_{0}\right) \frac{\partial \Phi_{2}}{\partial x_{0}}\left(\tau_{0}, \zeta_{0}\right)
$$

From the variational equation $\frac{d}{d t}\left(D_{X} \Phi\left(t, \zeta_{0}\right)\right)=\frac{\partial F}{\partial X}\left(\zeta_{0}\right) \frac{\partial \Phi}{\partial X}\left(t, \zeta_{0}\right)$, we have for all $0<$ $t \leq \tau_{0}$

$$
\left\{\begin{array}{l}
\frac{\partial \Phi_{1}\left(t, \zeta_{0}\right)}{\partial x_{0}}=\frac{d_{1}+u_{2}}{u_{2}-u_{1}} e^{u_{2} t}-\frac{d_{1}+u_{1}}{u_{2}-u_{1}} e^{u_{1} t}, \\
\frac{\partial \Phi_{2}\left(t, \zeta_{0}\right)}{\partial x_{0}}=\frac{a_{x}}{u_{2}-u_{1}}\left(e^{u_{2} t}-e^{u_{1} t}\right), \\
\frac{\partial \Phi_{1}\left(t, \zeta_{0}\right)}{\partial x_{1}}=-\frac{d_{1}+u_{2}}{u_{2}-u_{1}} \frac{d_{1}+u_{1}}{a_{x}}\left(e^{u_{2} t}-e^{u_{1} t}\right), \\
\frac{\partial \Phi_{2}\left(t, \zeta_{0}\right)}{\partial x_{1}}=-\frac{d_{1}+u_{1}}{u_{2}-u_{1}} e^{u_{2} t}+\frac{d_{1}+u_{2}}{u_{2}-u_{1}} e^{u_{1} t}, \\
\frac{\partial \Phi_{3}\left(t, \zeta_{0}\right)}{\partial y_{0}}=e^{\left(\gamma-a_{y}-\lambda \frac{a_{x}}{d_{1}} x_{0}^{*}\right) t}, \text { and } \\
\frac{\partial \Phi_{4}\left(t, \zeta_{0}\right)}{\partial y_{1}}=e^{-d_{2} t}
\end{array}\right.
$$

where $u_{1}=\frac{-\beta x_{0}^{*}-d_{1}-\sqrt{\Delta_{1}}}{2}<0, u_{2}=\frac{-\beta x_{0}^{*}-d_{1}+\sqrt{\Delta_{1}}}{2}<0$ and $\Delta_{1}=\left(\beta_{0} x_{0}^{*}-d_{1}\right)^{2}-$ $4 \lambda a_{x} x_{0}^{*}>0$ for either

$$
0<\beta-a_{x}<\frac{\left(\beta_{0} d_{1}+\lambda a_{x}\right)\left[\left(\beta_{0} d_{1}+2 \lambda a_{x}\right)-2 \sqrt{\lambda a_{x}\left(\beta_{0} d_{1}+\lambda a_{x}\right)}\right]}{\beta_{0}^{2} d_{1}}
$$

or

$$
\beta-a_{x}>\frac{\left(\beta_{0} d_{1}+\lambda a_{x}\right)\left[\left(\beta_{0} d_{1}+2 \lambda a_{x}\right)+2 \sqrt{\lambda a_{x}\left(\beta_{0} d_{1}+\lambda a_{x}\right)}\right]}{\beta_{0}^{2} d_{1}}
$$

(see Appendix, Subsection 4.1).

Then, we obtain $\Delta=\left(e^{u_{2} t}-e^{u_{1} t}\right)^{2}, \mu_{-}=e^{u_{1} \tau_{0}} \in(0,1)$ and $\mu_{+}=e^{u_{2} \tau_{0}} \in(0,1)$.

REMARK.

1) In the hypothesis ( $\mathrm{H} 1)$, the condition $\beta>a_{x}$ is a biological condition, which will allow the preservation of the population of healthy hematopoietic stem cells. On the other hand, $\beta<a_{x}+\frac{\left(\beta_{0} d_{1}+\lambda a_{x}\right)\left[\left(\beta_{0} d_{1}+2 \lambda a_{x}\right)-2 \sqrt{\lambda a_{x}\left(\beta_{0} d_{1}+\lambda a_{x}\right)}\right]}{\beta_{0}^{2} d_{1}}$ and

$\beta>a_{x}+\frac{\left(\beta_{0} d_{1}+\lambda a_{x}\right)\left[\left(\beta_{0} d_{1}+2 \lambda a_{x}\right)+2 \sqrt{\lambda a_{x}\left(\beta_{0} d_{1}+\lambda a_{x}\right)}\right]}{\beta_{0}^{2} d_{1}}$ are technical conditions which will make it possible to determine the stability of the periodic solutions without diseases.

2) If (H1) is satisfied and $\gamma>\gamma^{*}$ we have $T_{1}\left|\frac{\partial \Phi_{4}}{\partial y_{1}}\left(\tau_{0}, \zeta_{0}\right)\right|<1$ for $\tau_{0}<\tau_{0}^{*}$ and 
$T_{0}\left|\frac{\partial \Phi_{3}}{\partial y_{0}}\left(\tau_{0}, \zeta_{0}\right)\right|=1$ for $\tau_{0}=\tau_{0}^{*}$. That is we have a critical case at $\tau_{0}=\tau_{0}^{*}$.

3) From theorem 1.2, if (H1) is satisfied we show that in case of low growth rate of leukemic sensitive stem cells $\gamma\left(\leq \gamma^{*}\right)$ we can choose any period $\tau_{0}$ of treatment to have eradication of the disease. Otherwise, for high growth rate of leukemic sensitive stem cells $\gamma\left(>\gamma^{*}\right)$, the eradication of the disease is acquired only for period $\tau_{0}$ less than some threshold $\tau_{0}^{*}$.

\subsection{Bifurcation Analysis of nontrivial periodic solution}

In this subsection, we analyze the bifurcation of nontrivial periodic solutions of (5) (12) from $\zeta$ at $\tau_{0}=\tau_{0}^{*}$. This case is possible if $(H 1)$ is satisfied and $\gamma>\gamma^{*}$ (see theorem 1.2). The bifurcated solutions means that the disease is installed.

Let $\bar{\tau}$ and $\bar{X}$ such that $\tau=\tau_{0}^{*}+\bar{\tau}$ and $X=\zeta_{0}+\bar{X}$. The equation (15) is equivalent to

$$
M(\bar{\tau}, \bar{X})=0,
$$

where $M(\bar{\tau}, \bar{X})=\left(M_{1}(\bar{\tau}, \bar{X}), \ldots, M_{4}(\bar{\tau}, \bar{X})\right):=\zeta_{0}+\bar{X}-\Psi\left(\tau_{0}+\bar{\tau}, \zeta_{0}+\bar{X}\right)$. If $(\bar{\tau}, \bar{X})$ is a zero of $M$, then $\left(\zeta_{0}+\bar{X}\right)$ is a fixed point of $\Psi\left(\tau_{0}^{*}+\bar{\tau},.\right)$. Let

$$
D_{X} M(\bar{\tau}, \bar{X})=\left(\begin{array}{cccc}
a & b & c & d \\
e & f & \star & \star \\
\star & \star & g & \star \\
\star & \star & h & i
\end{array}\right) .
$$

For $(\bar{\tau}, \bar{X})=(0,(0,0,0,0))$, we have

$$
\begin{aligned}
D_{X} M(0,(0,0,0,0)) & =\left(\begin{array}{cccc}
a_{0} & b_{0} & c_{0} & d_{0} \\
e_{0} & f_{0} & \star & \star \\
\star & \star & g_{0} & \star \\
\star & \star & h_{0} & i_{0}
\end{array}\right) \\
& =\left(\begin{array}{cccc}
1-\frac{\partial \Phi_{1}}{\partial x_{0}} & -\frac{\partial \Phi_{1}}{\partial x_{1}} & -\frac{\partial \Phi_{1}}{\partial y_{0}} & -\frac{\partial \Phi_{1}}{\partial y_{1}} \\
-\frac{\partial \Phi_{2}}{\partial x_{0}} & 1-\frac{\partial \Phi_{2}}{\partial x_{1}} & 0 & 0 \\
0 & 0 & 1-T_{0} \frac{\partial \Phi_{3}}{\partial y_{0}} & 0 \\
0 & 0 & -T_{1} \frac{\partial \Phi_{4}}{\partial y_{0}} & 1-T_{1} \frac{\partial \Phi_{4}}{\partial y_{1}}
\end{array}\right)\left(\tau_{0}^{*}, \zeta_{0}\right) .
\end{aligned}
$$

Then $a_{0}=1-\frac{\partial \Phi_{1}}{\partial x_{0}}\left(\tau_{0}^{*}, \zeta_{0}\right), b_{0}=-\frac{\partial \Phi_{1}}{\partial x_{1}}\left(\tau_{0}^{*}, \zeta_{0}\right), c_{0}=-\frac{\partial \Phi_{1}}{\partial y_{0}}\left(\tau_{0}^{*}, \zeta_{0}\right), d_{0}=-\frac{\partial \Phi_{1}}{\partial y_{1}}$, $e_{0}=-\frac{\partial \Phi_{2}}{\partial x_{0}}\left(\tau_{0}^{*}, \zeta_{0}\right)$, 
$f_{0}=1-\frac{\partial \Phi_{2}}{\partial x_{1}}\left(\tau_{0}^{*}, \zeta_{0}\right), g_{0}=1-T_{0} \frac{\partial \Phi_{3}}{\partial y_{0}}\left(\tau_{0}^{*}, \zeta_{0}\right), h_{0}=-T_{1} \frac{\partial \Phi_{4}}{\partial y_{0}}\left(\tau_{0}^{*}, \zeta_{0}\right)$ and $i_{0}=1-$ $T_{1} \frac{\partial \Phi_{4}}{\partial y_{1}}\left(\tau_{0}^{*}, \zeta_{0}\right)$

We have the critical cases if and only if $\operatorname{det} D_{X} M(0,(0,0,0,0))=\left(a_{0} f_{0}-b_{0} e_{0}\right) g_{0} i_{0}=$ 0 . That is

$$
g_{0}=0
$$

since $i_{0}=1-T_{1} e^{-d_{2} \tau_{0}^{*}} \in(0,1)$ and $a_{0} f_{0}-b_{0} e_{0}=\left(1-e^{u_{2} \tau_{0}^{*}}\right)\left(1-e^{u_{1} \tau_{0}^{*}}\right) \in(0,1)$. We have $M(0,(0,0,0,0))=0$. Let $D_{X} M(0,(0,0,0,0))=E$, then $\operatorname{dim} \operatorname{ker}(E)=$ $\operatorname{codim} \mathcal{R}(\mathrm{E})=1$. Denote by $P_{1}$ and $P_{2}$ the projectors onto $\operatorname{ker}(E)$ and $\mathcal{R}(E)$ respectively, such that $P_{1}+P_{2}=I d_{\mathbf{R}^{4}}, P_{1} \mathbf{R}^{4}=\operatorname{span}\left\{\mathrm{Y}_{0}\right\}=\operatorname{ker}(\mathrm{E})$, with $Y_{0}=\left(q_{1}, q_{2}, 1, q_{4}\right)$, $q_{1}=\frac{f_{0}\left(c_{0} i_{0}-d_{0} h_{0}\right)}{i_{0}\left(b_{0} e_{0}-a_{0} f_{0}\right)}, q_{2}=-\frac{e_{0}\left(c_{0} i_{0}-d_{0} h_{0}\right)}{i_{0}\left(b_{0} e_{0}-a_{0} f_{0}\right)}, q_{4}=\frac{-h_{0}}{i_{0}}$ and $P_{2} \mathbf{R}^{4}=\operatorname{span}\{(1,0,0,0),(0,1,0,0),(0,0,0,1)\}=\mathcal{R}(E)$.

Then $\left(I-P_{1}\right) \mathbf{R}^{4}=\operatorname{span}\{(1,0,0,0),(0,1,0,0),(0,0,0,1)\}$ and $\left(I-P_{2}\right) \mathbf{R}^{4}=\operatorname{span}\{(0,0,1,0)\}$.

Equation (19) is equivalent to

$$
\left\{\begin{array}{l}
M_{1}\left(\bar{\tau}, \sigma Y_{0}+Z\right)=0 \\
M_{2}\left(\bar{\tau}, \sigma Y_{0}+Z\right)=0 \\
M_{3}\left(\bar{\tau}, \sigma Y_{0}+Z\right)=0 \\
M_{4}\left(\bar{\tau}, \sigma Y_{0}+Z\right)=0
\end{array}\right.
$$

where $Z=\left(z_{1}, z_{2}, 0, z_{4}\right),(\bar{\tau}, \bar{X})=\left(\bar{\tau}, \sigma Y_{0}+Z\right)$ and $\left(\sigma, z_{1}, z_{2}, z_{4}\right) \in \mathbf{R}^{4}$.

From the three equations of (22), we have

$$
\begin{array}{rll}
\operatorname{det}\left(\begin{array}{ccc}
\frac{\partial M_{1}(0,(0,0,0,0))}{\partial z_{1}} & \frac{\partial M_{1}(0,(0,0,0,0))}{\partial z_{2}} & \frac{\partial M_{1}(0,(0,0,0,0))}{\partial z_{4}} \\
\frac{\partial M_{2}(0,(0,0,0,0))}{\left.\partial z_{1}, 0,0\right)} & \frac{\partial M_{2}(0,(0,0,0,0))}{\partial z_{2}} & \frac{\partial M_{2}(0,(0,0,0,0))}{\partial z_{4}} \\
\frac{\partial M_{4}(0,(0,0,0,0))}{\partial z_{1}} & \frac{\partial M_{4}(0,(0,0,0,0))}{\partial z_{2}} & \frac{\partial M_{4}(0,(0,0,0,0))}{\partial z_{4}}
\end{array}\right)=\operatorname{det}\left(\begin{array}{ccc}
a_{0} & b_{0} & d_{0} \\
e_{0} & f_{0} & 0 \\
0 & 0 & i_{0}
\end{array}\right) \\
=i_{0}\left(a_{0} f_{0}-e_{0} b_{0}\right) \neq 0 .
\end{array}
$$

From the implicit function theorem, there exist a unique continuous function $Z^{*}$, such that

$$
\begin{aligned}
Z^{*}(\bar{\tau}, \sigma)= & \left(z_{1}^{*}(\bar{\tau}, \sigma), z_{2}^{*}(\bar{\tau}, \sigma), 0, z_{4}^{*}(\bar{\tau},)\right), Z^{*}(0,0)=(0,0,0,0) \text { and } \\
& M_{i}\left(\bar{\tau},\left(q_{1} \sigma+z_{1}^{*}(\bar{\tau}, \sigma), q_{2} \sigma+z_{2}^{*}(\bar{\tau}, \sigma), \sigma, q_{4} \sigma+z_{4}^{*}(\bar{\tau}, \sigma)\right)\right)=0,
\end{aligned}
$$

for $i=1,2,4$, with $\sigma$ and $\bar{\tau}$ small enough.

We have $\frac{\partial Z^{*}}{\partial \bar{\tau}}(0,0)=0$ and $\frac{\partial Z^{*}}{\partial \sigma}(0,0)=\left(-\frac{f_{0} c_{0}}{a_{0} f_{0}-e_{0} b_{0}}, \frac{c_{0} e_{0}}{a_{0} f_{0}-e_{0} b_{0}}, 0,0\right)$ (see Appendix, subsection 4.3).

We have the following theorem.

Theorem 1.3 Let (H1) be satisfied and $\gamma>\gamma^{*}$. There exist $\lambda_{0}>0$ such that for $\lambda \in$ $\left(0, \lambda_{0}\right)$ we have a supercritical bifurcation of nontrivial periodic solutions of (5)-(12) with period $\tau(\sigma)=\tau_{0}^{*}+\bar{\tau}(\sigma)$ starting from $\zeta_{0}+\sigma Y_{0}+Z^{*}(\bar{\tau}(\sigma), \sigma)$ for $\sigma(>0)$ small enough where $\bar{\tau}(\sigma)=-\frac{\mathcal{C}}{2 \mathcal{B}} \sigma+\circ(\sigma)$.

Proof. -

We have $M(\bar{\tau}, \bar{X})=0$ if and only if

$$
\omega(\bar{\tau}, \sigma)=M_{3}\left(\bar{\tau},\left(q_{1} \sigma+z_{1}^{*}(\bar{\tau}, \sigma), q_{2} \sigma+z_{2}^{*}(\bar{\tau}, \sigma), \sigma, q_{4} \sigma+z_{4}^{*}(\bar{\tau}, \sigma)\right)\right)=0 .
$$


We find $\omega(0,0)=0$ and $\frac{\partial \omega(0,0)}{\partial \bar{\tau}}=\frac{\partial \omega(0,0)}{\partial \sigma}=0$ (see Appendix, subsection 4.4).

Let $\mathcal{A}=\frac{\partial^{2} \omega(0,0)}{\partial \bar{\tau}^{2}}, \mathcal{B}=\frac{\partial^{2} \omega(0,0)}{\partial \bar{\tau} \partial \sigma}$ and $\mathcal{C}=\frac{\partial^{2} \omega(0,0)}{\partial \sigma^{2}}$. It's shown that $\mathcal{A}=0$ (see Appendix, subsection 4.5). Hence

$$
\omega(\bar{\tau}, \sigma)=\mathcal{B} \bar{\tau} \sigma+\mathcal{C} \frac{\sigma^{2}}{2}+o\left(|\sigma|^{2}+|\bar{\tau}|^{2}\right)
$$

where

$$
\begin{aligned}
\mathcal{B} & =-\frac{\partial \Theta_{3}}{\partial y_{0}} \frac{\partial^{2} \Phi_{3}\left(\tau_{0}, \zeta_{0}\right)}{\partial \bar{\tau} \partial y_{0}} \\
& =-\left(\gamma-a_{y}-\lambda \frac{a_{x}}{d_{1}} x_{0}^{*}\right)<0
\end{aligned}
$$

and

$$
\begin{aligned}
\mathcal{C}= & -\frac{\partial \Theta_{3}}{\partial y_{0}}\left\{2 \frac{\partial^{2} \Phi_{3}\left(\tau_{0}, \zeta_{0}\right)}{\partial x_{0} \partial y_{0}}\left(q_{1}+\frac{\partial z_{1}^{*}(0,0)}{\partial \sigma}\right)+2 \frac{\partial^{2} \Phi_{3}\left(\tau_{0}, \zeta_{0}\right)}{\partial x_{1} \partial y_{0}}\left(q_{2}+\frac{\partial z_{2}^{*}(0,0)}{\partial \sigma}\right)\right. \\
& \left.+2 \frac{\partial^{2} \Phi_{3}\left(\tau_{0}, \zeta_{0}\right)}{\partial y_{0} \partial y_{1}} q_{4}+\frac{\partial^{2} \Phi_{3}\left(\tau_{0}, \zeta_{0}\right)}{\partial y_{0}^{2}}\right\} \\
= & 2 \lambda \frac{a_{x}}{u_{2}-u_{1}}\left(\frac{e^{u_{2} \tau_{0}}-1}{u_{2}}-\frac{e^{u_{1} \tau_{0}}-1}{u_{1}}\right) \frac{f_{0}\left(d_{0} h_{0}-2 c_{0} i_{0}\right)}{i_{0}\left(a_{0} f_{0}-b_{0} e_{0}\right)} \\
& +2 \lambda\left(\frac{d_{1}+u_{2}}{u_{2}-u_{1}}\left(\frac{e^{u_{2} \tau_{0}}-1}{u_{2}}\right)-\frac{d_{1}+u_{1}}{u_{2}-u_{1}}\left(\frac{e^{u_{1} \tau_{0}}-1}{u_{1}}\right)\right) \frac{e_{0}\left(d_{0} h_{0}-2 c_{0} i_{0}\right)}{i_{0}\left(a_{0} f_{0}-b_{0} e_{0}\right)} \\
& +2 \lambda \alpha\left(\frac{e^{-d_{2} \tau_{0}}-1}{d_{2}}\right) \frac{h_{0}}{i_{0}}+2 \gamma_{0}\left(\frac{e^{\left(\gamma-a_{y}-\lambda \frac{a_{x}}{d_{1}} x_{0}^{*}\right) \tau_{0}}-1}{\gamma-a_{y}-\lambda \frac{a_{x}}{d_{1}} x_{0}^{*}}\right) \\
& +\lambda \alpha \frac{a_{y}}{\gamma-a_{y}-\lambda \frac{a_{x}}{d_{1}} x_{0}^{*}+d_{2}}\left(\frac{e^{\left(\gamma-a_{y}-\lambda \frac{a_{x}}{d_{1}} x_{0}^{*}\right) \tau_{0}}-1}{\gamma-a_{y}-\lambda \frac{a_{x}}{d_{1}} x_{0}^{*}}+\frac{e^{-d_{2} \tau_{0}}-1}{d_{2}}\right) .
\end{aligned}
$$

For $\lambda=0$, we have

$$
\mathcal{C}=2 \gamma_{0}\left(\frac{e^{\left(\gamma-a_{y}\right) \tau_{0}}-1}{\gamma-a_{y}}\right)>0
$$

REMARK. -

From theorem 1.3, we deduce that for high growth rate of leukemic sensitive stem cells $\gamma\left(>\gamma^{*}\right)$ and period of treatment dose $\tau_{0}=\tau_{0}^{*}$ there is lost of stability of the disease free equilibrium and we note the presence of nontrivial periodic solution which means that the disease is installed for period $\tau_{0}(\sigma)$ close to $\tau_{0}^{*}$.

\section{Numerical simulations}

To illustrate our results, we give some numerical simulations. 
In Figure 1, we consider the case of the theorem 1.2, we have the stability of the healthy steady state $E_{f}$.
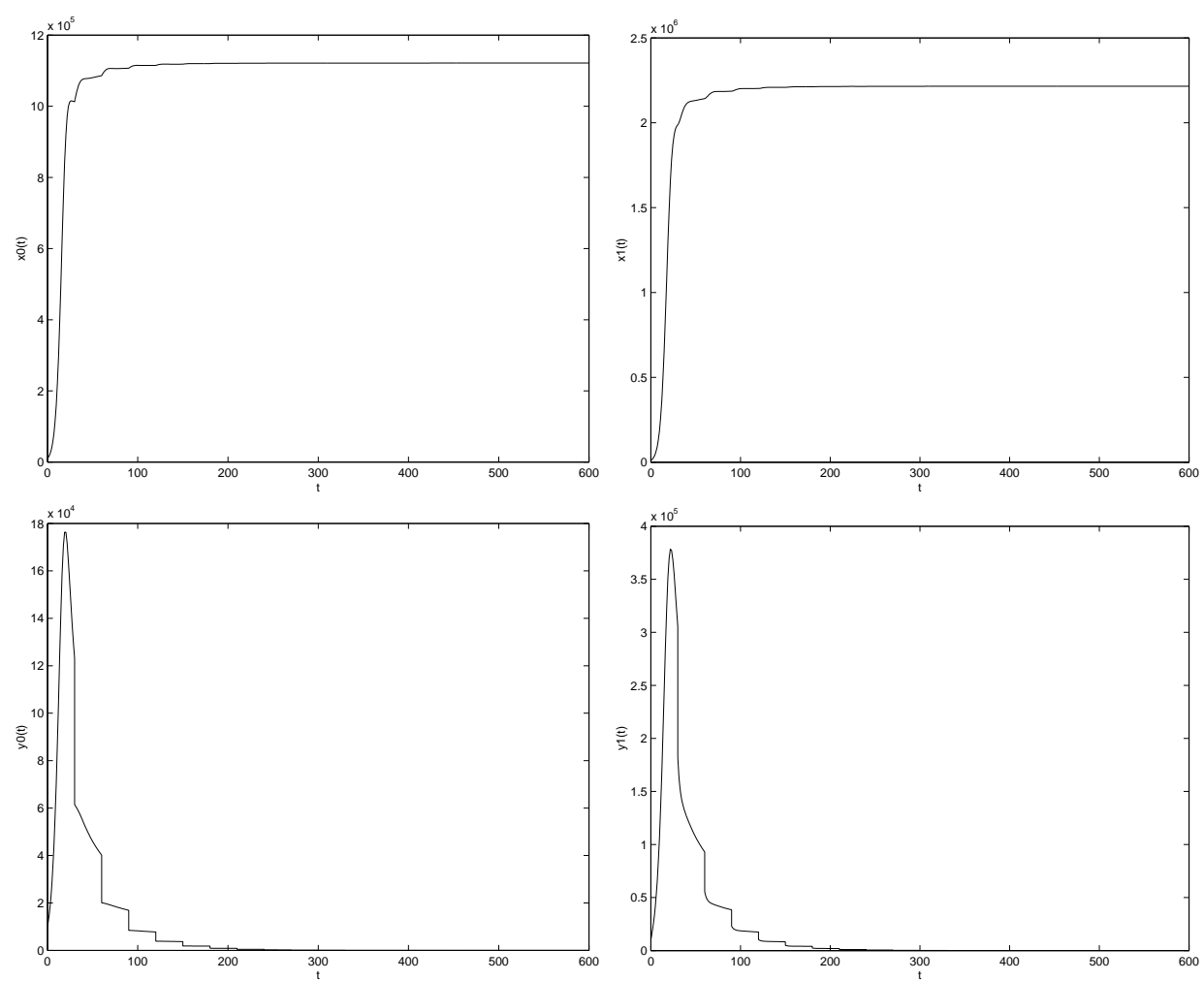

Figure 1. The curves of normal stem cells (top left), normal progenitor cells (top right), leukemic stem cells (bottom left) and leukemic progenitor cells (bottom right) with $\beta=1.1$, $a_{x}=0.8, \beta_{0}=0.00000007, \lambda=0.0000001, d_{1}=0.405, \gamma=1.121, a_{y}=0.9, \gamma_{0}=$ $0.0000003, \alpha=0.8, d_{2}=0.402, T_{0}=0.5, T_{1}=0.6, \tau=30, x_{0}(0)=10000, x_{1}(0)=10000$, $y_{0}(0)=10000$ and $y_{1}(0)=10000$ 
In the Figure 2, we consider the case of the theorem 1.2, we have the instability of the healthy steady state $E_{f}$, we see that leukemic cells reappears.
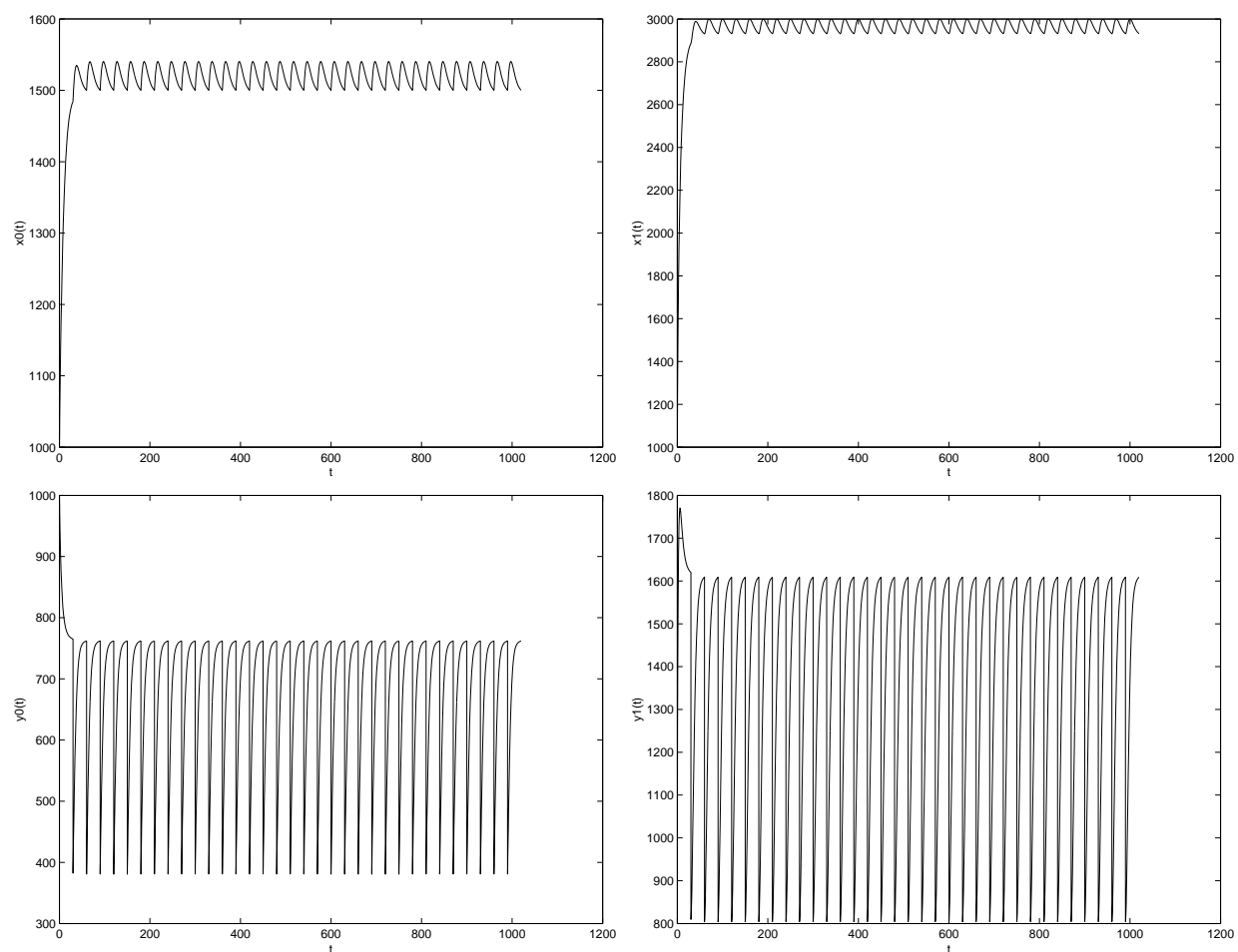

Figure 2. The curves of normal stem cells (top left), normal progenitor cells (top right), leukemic stem cells (bottom left) and leukemic progenitor cells (bottom right) with $\beta=0.95$, $a_{x}=0.8, \beta_{0}=0.00007, \lambda=0.0001, d_{1}=0.41, \gamma=1.121, a_{y}=0.85, \gamma_{0}=0.0003, \alpha=0.8$, $d_{2}=0.402, T_{0}=0.5, T_{1}=0.5, \tau=30, x_{0}(0)=1000, x_{1}(0)=1000, y_{0}(0)=1000$ and $y_{1}(0)=1000$ 
In Figure 3, we consider the case of the theorem 1.3, we have the bifurcation of periodic solutions for the treatment period $\tau=\tau_{0}^{*}$.
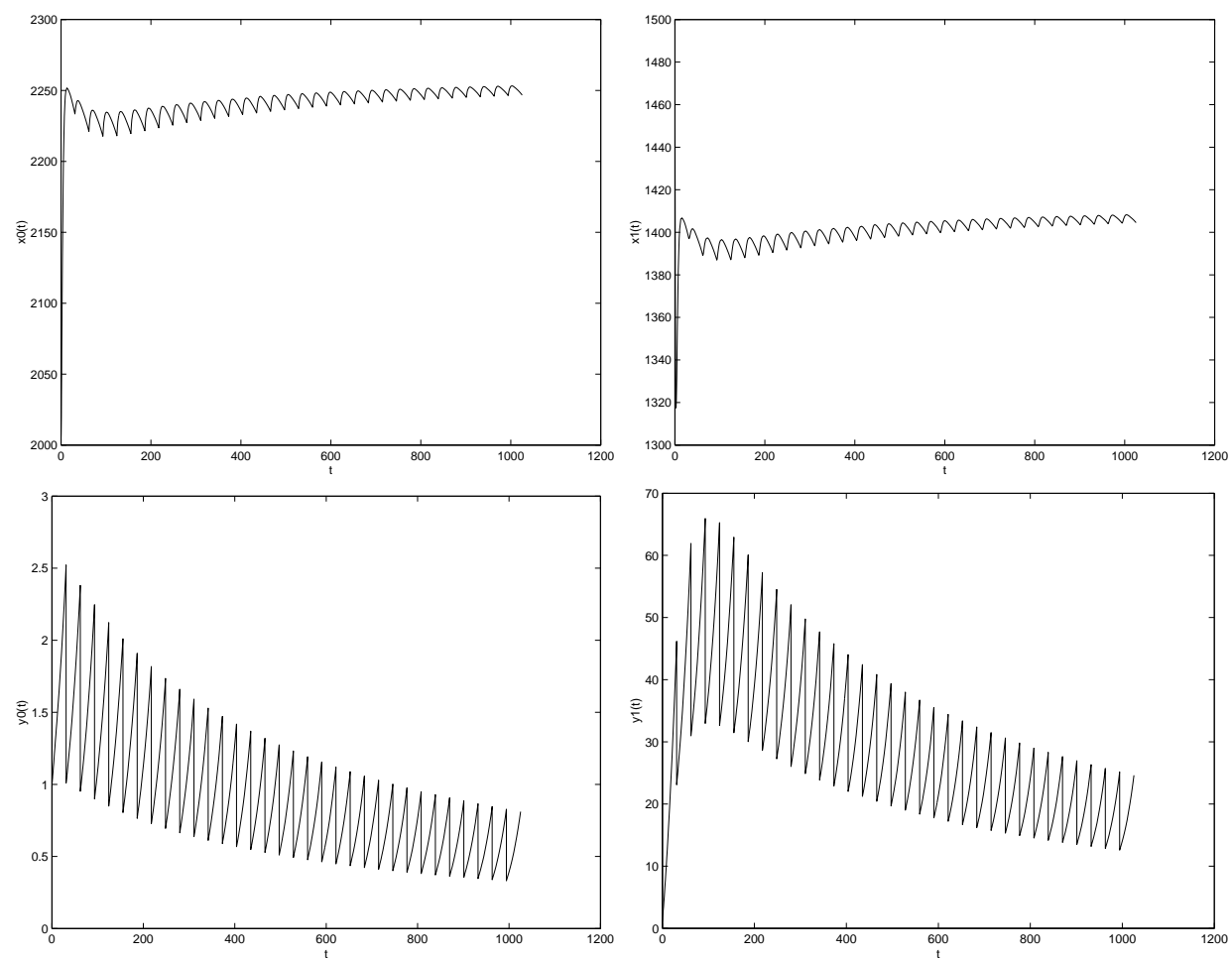

Figure 3. The curves of normal stem cells (top left), normal progenitor cells (top right), leukemic stem cells (bottom left) and leukemic progenitor cells (bottom right) with $\beta=0.8$, $a_{x}=0.5, \beta_{0}=0.00007, \lambda=0.0001, d_{1}=0.8, \gamma=1.121, a_{y}=0.95, \gamma_{0}=0.0003, \alpha=0.8$, $d_{2}=0.007, T_{0}=0.4, T_{1}=0.5, \tau=\tau_{0}^{*}=31.0706, x_{0}(0)=2000, x_{1}(0)=1500, y_{0}(0)=1$ and $y_{1}(0)=1$

\section{Conclusions}

In this work we have analyzed a mathematical model of chronic myeloid leukemia (CML) which is an extension of a model developed in [9] in the case without medical treatment. In our work, we considered the case of a treatment with instantaneous effect described by discrete equations called impulses. We have studied the stability of the healthy equilibrium (trivial solution), it becomes stable if the growth rate of resistant stem cells $\gamma$ does not exceed a certain threshold $\gamma^{*}$, if it reaches this threshold we obtain a critical case which gives bifurcations what we want say that is the tumor persists and remains viable. 


\section{Appendix}

\subsection{First derivatives of $\Phi$}

For all $t \in(0, \tau]$, we have $\frac{d}{d t} D_{X}\left(\Phi\left(t, \zeta_{0}\right)\right)=\frac{\partial F}{\partial X}\left(\zeta_{0}\right) \frac{\partial \Phi}{\partial X}\left(t, \zeta_{0}\right)$ with the initial condition $D_{X}\left(\Phi\left(0, \zeta_{0}\right)\right)=I_{\mathbf{R}^{4}}$, where

$$
\begin{aligned}
& \frac{d}{d t} D_{X}\left(\Phi\left(t, \zeta_{0}\right)\right)=\frac{d}{d t}\left(\begin{array}{cccc}
\frac{\partial \Phi_{1}\left(t, \zeta_{0}\right)}{\partial x_{0}} & \frac{\partial \Phi_{1}\left(t, \zeta_{0}\right)}{\partial x_{1}} & \frac{\partial \Phi_{1}\left(t, \zeta_{0}\right)}{\partial y_{0}} & \frac{\partial \Phi_{1}\left(t, \zeta_{0}\right)}{\partial y_{1}} \\
\frac{\partial \Phi_{2}\left(t, \zeta_{0}\right)}{\partial x_{0}} & \frac{\partial \Phi_{2}\left(t, \zeta_{0}\right)}{\partial x_{1}} & \frac{\partial \Phi_{2}\left(t, \zeta_{0}\right)}{\partial y_{0}} & \frac{\partial \Phi_{2}\left(t, \zeta_{0}\right)}{\partial y_{1}} \\
\frac{\partial \Phi_{3}\left(t, \zeta_{0}\right)}{\partial x_{0}} & \frac{\partial \Phi_{3}\left(t, \zeta_{0}\right)}{\partial x_{1}} & \frac{\partial \Phi_{3}\left(t, \zeta_{0}\right)}{\partial y_{0}} & \frac{\partial \Phi_{3}\left(t, \zeta_{0}\right)}{\partial y_{1}} \\
\frac{\partial \Phi_{4}\left(t, \zeta_{0}\right)}{\partial x_{0}} & \frac{\partial \Phi_{4}\left(t, \zeta_{0}\right)}{\partial x_{1}} & \frac{\partial \Phi_{4}\left(t, \zeta_{0}\right)}{\partial y_{0}} & \frac{\partial \Phi_{4}\left(t, \zeta_{0}\right)}{\partial y_{1}}
\end{array}\right), \\
& \begin{aligned}
\frac{\partial F}{\partial X}\left(\zeta_{0}\right) & =\left(\begin{array}{cccc}
\frac{\partial F_{1}(\zeta(t))}{\left.\partial x_{0}(t)\right)} & \frac{\partial F_{1}(\zeta(t))}{\partial x_{1}(t)} & \frac{\partial F_{1}(\zeta(t))}{\partial y_{0}(t)} & \frac{\partial F_{1}(\zeta(t))}{\partial y_{1}(t)} \\
\frac{\partial F_{2}(\zeta(t))}{\left.\partial x_{0}(t)\right)} & \frac{\partial F_{2}(\zeta(t))}{\partial x_{1}(t)} & \frac{\partial F_{2}(\zeta(t))}{\partial y_{0}(t)} & \frac{\partial F_{2}(\zeta(t))}{\partial y_{1}(t)} \\
\frac{\partial F_{3}(\zeta(t))}{\left.\partial x_{0}(t)\right)} & \frac{\partial F_{3}(\zeta(t))}{\partial x_{1}(t)} & \frac{\partial F_{3}(\zeta(t))}{\partial y_{0}} & \frac{\partial F_{3}(\zeta(t))}{\partial y_{1}} \\
\frac{\partial F_{4}(\zeta(t))}{\partial x_{0}} & \frac{\partial F_{4}(\zeta(t))}{\partial x_{1}} & \frac{\partial F_{4}(\zeta(t))}{\partial y_{0}} & \frac{\partial F_{4}(\zeta(t))}{\partial y_{1}}
\end{array}\right) \\
& =\left(\begin{array}{cccc}
-\beta_{0} x_{0}^{*} & -\lambda x_{0}^{*} & 0 & -\lambda x_{0}^{*} \\
a_{x} & -d_{1} & 0 & 0 \\
0 & 0 & \gamma-a_{y}-\lambda \frac{a_{x}}{d_{1}} x_{0}^{*} & 0 \\
0 & 0 & a_{y} & -d_{2}
\end{array}\right),
\end{aligned}
\end{aligned}
$$

and

$$
\frac{\partial \Phi}{\partial X}\left(t, \zeta_{0}\right)=\left(\begin{array}{cccc}
\frac{\partial \Phi_{1}\left(t, \zeta_{0}\right)}{\partial x_{0}} & \frac{\partial \Phi_{1}\left(t, \zeta_{0}\right)}{\partial x_{1}} & \frac{\partial \Phi_{1}\left(t, \zeta_{0}\right)}{\partial y_{0}} & \frac{\partial \Phi_{1}\left(t, \zeta_{0}\right)}{\partial y_{1}} \\
\frac{\partial \Phi_{2}\left(t, \zeta_{0}\right)}{\partial x_{0}} & \frac{\partial \Phi_{2}\left(t, \zeta_{0}\right)}{\partial x_{1}} & \frac{\partial \Phi_{2}\left(t, \zeta_{0}\right)}{\partial y_{0}} & \frac{\partial \Phi_{2}\left(t, \zeta_{0}\right)}{\partial y_{1}} \\
\frac{\partial \Phi_{3}\left(t, \zeta_{0}\right)}{\partial x_{0}} & \frac{\partial \Phi_{3}\left(t, \zeta_{0}\right)}{\partial x_{1}} & \frac{\partial \Phi_{3}\left(t, \zeta_{0}\right)}{\partial y_{0}} & \frac{\partial \Phi_{3}\left(t, \zeta_{0}\right)}{\partial y_{1}} \\
\frac{\partial \Phi_{4}\left(t, \zeta_{0}\right)}{\partial x_{0}} & \frac{\partial \Phi_{4}\left(t, \zeta_{0}\right)}{\partial x_{1}} & \frac{\partial \Phi_{4}\left(t, \zeta_{0}\right)}{\partial y_{0}} & \frac{\partial \Phi_{4}\left(t, \zeta_{0}\right)}{\partial y_{1}}
\end{array}\right) .
$$

From Cauchy Lipschitz theorem (uniqueness of solution) we obtain that $\frac{\partial \Phi_{2}\left(t, \zeta_{0}\right)}{\partial y_{i}}=$ $\frac{\partial \Phi_{3}\left(t, \zeta_{0}\right)}{\partial x_{i}}=\frac{\partial \Phi_{4}\left(t, \zeta_{0}\right)}{\partial x_{i}}=0, i \in\{0,1\}$ and $\frac{\partial \Phi_{3}\left(t, \zeta_{0}\right)}{\partial y_{1}}=0$. Moreover, we have

$$
\begin{aligned}
\frac{d}{d t}\left(\frac{\partial \Phi_{1}\left(t, \zeta_{0}\right)}{\partial x_{0}}\right)= & \frac{\partial F_{1}(\zeta(t))}{\partial x_{0}} \frac{\partial \Phi_{1}\left(t, \zeta_{0}\right)}{\partial x_{0}}+\frac{\partial F_{1}(\zeta(t))}{\partial x_{1}} \frac{\partial \Phi_{2}\left(t, \zeta_{0}\right)}{\partial x_{0}}, \\
\frac{d}{d t}\left(\frac{\partial \Phi_{1}\left(t, \zeta_{0}\right)}{\partial x_{1}}\right)= & \frac{\partial F_{1}(\zeta(t))}{\partial x_{0}} \frac{\partial \Phi_{1}\left(t, \zeta_{0}\right)}{\partial x_{1}}+\frac{\partial F_{1}(\zeta(t))}{\partial x_{1}} \frac{\partial \Phi_{2}\left(t, \zeta_{0}\right)}{\partial x_{1}} \\
\frac{d}{d t}\left(\frac{\partial \Phi_{1}\left(t, \zeta_{0}\right)}{\partial y_{0}}\right)= & \frac{\partial F_{1}(\zeta(t))}{\partial x_{0}} \frac{\partial \Phi_{1}\left(t, \zeta_{0}\right)}{\partial y_{0}}+\frac{\partial F_{1}(\zeta(t))}{\partial y_{1}} \frac{\partial \Phi_{4}\left(t, \zeta_{0}\right)}{\partial y_{0}}, \\
\frac{d}{d t}\left(\frac{\partial \Phi_{1}\left(t, \zeta_{0}\right)}{\partial y_{1}}\right)= & \frac{\partial F_{1}(\zeta(t))}{\partial x_{0}} \frac{\partial \Phi_{1}\left(t, \zeta_{0}\right)}{\partial y_{1}}+\frac{\partial F_{1}(\zeta(t))}{\partial y_{1}} \frac{\partial \Phi_{4}\left(t, \zeta_{0}\right)}{\partial y_{1}} \\
\frac{d}{d t}\left(\frac{\partial \Phi_{2}\left(t, \zeta_{0}\right)}{\left.\partial x_{0}\right)}=\right. & \frac{\partial F_{2}(\zeta(t))}{\partial x_{0}} \frac{\partial \Phi_{1}\left(t, \zeta_{0}\right)}{\partial x_{0}}+\frac{\partial F_{2}(\zeta(t))}{\partial x_{1}} \frac{\partial \Phi_{2}\left(t, \zeta_{0}\right)}{\partial x_{0}} \\
\frac{d}{d t}\left(\frac{\partial \Phi_{2}\left(t, \zeta_{0}\right)}{\partial x_{1}}\right)= & \frac{\partial F_{2}(\zeta(t))}{\partial x_{0}} \frac{\partial \Phi_{1}\left(t, \zeta_{0}\right)}{\partial x_{1}}+\frac{\partial F_{2}(\zeta(t))}{\partial x_{1}} \frac{\partial \Phi_{2}\left(t, \zeta_{0}\right)}{\partial x_{1}}, \\
\frac{d}{d t}\left(\frac{\partial \Phi_{3}\left(t, \zeta_{0}\right)}{\partial y_{0}}\right)= & \frac{\partial F_{3}(\zeta(t))}{\partial y_{0}} \frac{\partial \Phi_{3}\left(t, \zeta_{0}\right)}{\partial y_{0}}, \\
\frac{d}{d t}\left(\frac{\partial \Phi_{4}\left(t, \zeta_{0}\right)}{\left.\partial y_{0}\right)}=\right. & \frac{\partial F_{4}(\zeta(t))}{\partial y_{0}} \frac{\partial \Phi_{3}\left(t, \zeta_{0}\right)}{\partial y_{0}}+\frac{\partial F_{4}(\zeta(t))}{\partial y_{1}} \frac{\partial \Phi_{4}\left(t, \zeta_{0}\right)}{\partial y_{0}}
\end{aligned}
$$




$$
\frac{d}{d t}\left(\frac{\partial \Phi_{4}\left(t, \zeta_{0}\right)}{\partial y_{1}}\right)=\frac{\partial F_{4}(\zeta(t))}{\partial y_{1}} \frac{\partial \Phi_{4}\left(t, \zeta_{0}\right)}{\partial y_{1}}
$$

From (31) we obtain $\frac{\partial \Phi_{3}\left(t, \zeta_{0}\right)}{\partial y_{0}}=e^{\left(\gamma-a_{y}-\lambda \frac{a_{x}}{d_{1}} x_{0}^{*}\right) t}$.

From (32) we obtain $\frac{\partial \Phi_{4}\left(t, \zeta_{0}\right)}{\partial y_{0}}=\frac{a_{y}}{\gamma-a_{y}-\lambda \frac{a_{x}}{d_{1}} x_{0}^{*}+d_{2}}\left(e^{\left(\gamma-a_{y}-\lambda \frac{a_{x}}{d_{1}} x_{0}^{*}\right) t}-e^{-d_{2} t}\right)$.

From (33) we have $\frac{\partial \Phi_{4}\left(t, \zeta_{0}\right)}{\partial y_{1}}=e^{-d_{2} t}$.

From (25) and (29) we have $\left(\begin{array}{c}\frac{\partial \Phi_{1}\left(t, \zeta_{0}\right)}{\partial x_{0}} \\ \frac{\partial \Phi_{2}\left(t, \zeta_{0}\right)}{\partial x_{0}}\end{array}\right)=e^{t A}\left(\begin{array}{c}\frac{\partial \Phi_{1}\left(0, \zeta_{0}\right)}{\partial x_{0}} \\ \frac{\partial \Phi_{2}\left(0, \zeta_{0}\right)}{\partial x_{0}}\end{array}\right)=e^{t A}\left(\begin{array}{l}1 \\ 0\end{array}\right)$, where

$$
\begin{gathered}
A=\left(\begin{array}{cc}
\frac{\partial F_{1}(\zeta(t))}{\partial x_{0}(t)} & \frac{\partial F_{1}(\zeta(t))}{\partial x_{1}(t)} \\
\frac{\partial F_{2}(\zeta(t))}{\partial x_{0}} & \frac{\partial F_{2}(\zeta(t)}{\partial x_{1}}
\end{array}\right)=\left(\begin{array}{cc}
-\beta_{0} x_{0}^{*} & -\lambda x_{0}^{*} \\
a_{x} & -d_{1}
\end{array}\right)=P V P^{-1}, V=\left(\begin{array}{cc}
u_{1} & 0 \\
0 & u_{2}
\end{array}\right), \\
P=\left(\begin{array}{cc}
\frac{d_{1}+u_{1}}{a_{x}} & \frac{d_{1}+u_{2}}{a_{x}} \\
1 & 1
\end{array}\right), P^{-1}=\left(\begin{array}{cc}
-\frac{a_{x}}{u_{2}-u_{1}} & \frac{d_{1}+u_{2}}{u_{2}-u_{1}} \\
\frac{a_{x}}{u_{2}-u_{1}} & -\frac{d_{1}+u_{1}}{u_{2}-u_{1}}
\end{array}\right),
\end{gathered}
$$

where $u_{1}=\frac{\beta x_{0}^{*}+d_{1}-\sqrt{\Delta}}{2}, u_{2}=\frac{\beta x_{0}^{*}+d_{1}+\sqrt{\Delta}}{2}, \Delta=\left(\beta_{0} x_{0}^{*}-d_{1}\right)^{2}-4 \lambda a_{x} x_{0}^{*}$ and

$$
\begin{aligned}
& e^{t A}=P e^{t V} P^{-1} \\
& =\left(\begin{array}{cc}
\frac{d_{1}+u_{1}}{a_{x}} & \frac{d_{1}+u_{2}}{a_{x}} \\
1 & 1
\end{array}\right)\left(\begin{array}{cc}
e^{u_{1} t} & 0 \\
0 & e^{u_{2} t}
\end{array}\right)\left(\begin{array}{cc}
-\frac{a_{x}}{u_{2}-u_{1}} & \frac{d_{1}+u_{2}}{u_{2}-u_{1}} \\
\frac{a_{x}}{u_{2}-u_{1}} & -\frac{d_{1}+u_{1}}{u_{2}-u_{1}}
\end{array}\right) \\
& =\left(\begin{array}{cc}
\frac{d_{1}+u_{1}}{a_{x}} e^{u_{1} t} & \frac{d_{1}+u_{2}}{a_{x}} e^{u_{2} t} \\
e^{u_{1} t} & e^{u_{2} t}
\end{array}\right)\left(\begin{array}{cc}
-\frac{a_{x}}{u_{2}-u_{1}} & \frac{d_{1}+u_{2}}{u_{2}-u_{1}} \\
\frac{a_{x}}{u_{2}-u_{1}} & -\frac{d_{1}+u_{1}}{u_{2}-u_{1}}
\end{array}\right) \\
& =\left(\begin{array}{cc}
\frac{d_{1}+u_{2}}{u_{2}-u_{1}} e^{u_{2} t}-\frac{d_{1}+u_{1}}{u_{2}-u_{1}} e^{u_{1} t} & -\frac{d_{1}+u_{2}}{u_{2}-u_{1}} \frac{d_{1}+u_{1}}{a_{x}}\left(e^{u_{2} t}-e^{u_{1} t}\right) \\
\frac{a_{x}}{u_{2}-u_{1}}\left(e^{u_{2} t}-e^{u_{1} t}\right) & -\frac{d_{1}+u_{1}}{u_{2}-u_{1}} e^{u_{2} t}+\frac{d_{1}+u_{2}}{u_{2}-u_{1}} e^{u_{1} t}
\end{array}\right) .
\end{aligned}
$$

We obtain

$$
\left\{\begin{aligned}
\frac{\partial \Phi_{1}\left(t, \zeta_{0}\right)}{\partial x_{0}} & =\frac{d_{1}+u_{2}}{u_{2}-u_{1}} e^{u_{2} t}-\frac{d_{1}+u_{1}}{u_{2}-u_{1}} e^{u_{1} t} \\
\frac{\partial \Phi_{2}\left(t, \zeta_{0}\right)}{\partial x_{0}} & =\frac{a_{x}}{u_{2}-u_{1}}\left(e^{u_{2} t}-e^{u_{1} t}\right)
\end{aligned}\right.
$$

From (26) and (30) we have $\left(\begin{array}{c}\frac{\partial \Phi_{1}\left(t, \zeta_{0}\right)}{\partial x_{1}} \\ \frac{\partial \Phi_{2}\left(t, \zeta_{0}\right)}{\partial x_{1}}\end{array}\right)=e^{t A}\left(\begin{array}{c}\frac{\partial \Phi_{1}\left(0, \zeta_{0}\right)}{\partial x_{1}} \\ \frac{\partial \Phi_{2}\left(0, \zeta_{0}\right)}{\partial x_{1}}\end{array}\right)=e^{t A}\left(\begin{array}{l}0 \\ 1\end{array}\right)$. We obtain

$$
\left\{\begin{array}{l}
\frac{\partial \Phi_{1}\left(t, \zeta_{0}\right)}{\partial x_{1}}=-\frac{d_{1}+u_{2}}{u_{2}-u_{1}} \frac{d_{1}+u_{1}}{a_{x}}\left(e^{u_{2} t}-e^{u_{1} t}\right), \\
\frac{\partial \Phi_{2}\left(t, \zeta_{0}\right)}{\partial x_{1}}=-\frac{d_{1}+u_{1}}{u_{2}-u_{1}} e^{u_{2} t}+\frac{d_{1}+u_{2}}{u_{2}-u_{1}} e^{u_{1} t}
\end{array}\right.
$$

From (27) we have

$$
\frac{\partial \Phi_{1}\left(t, \zeta_{0}\right)}{\partial y_{0}}=-\frac{\lambda a_{y} x_{0}^{*} e^{-\beta_{0} x_{0}^{*} t}}{\gamma-a_{y}-\lambda \frac{a_{x}}{d_{1}} x_{0}^{*}+d_{2}}\left(\frac{e^{\left(\gamma-a_{y}-\lambda \frac{a_{x}}{d_{1}} x_{0}^{*}+\beta_{0} x_{0}^{*}\right) t}-1}{\gamma-a_{y}-\lambda \frac{a_{x}}{d_{1}} x_{0}^{*}+\beta_{0} x_{0}^{*}}-\frac{e^{\left(\beta_{0} x_{0}^{*}-d_{2}\right) t}-1}{\beta_{0} x_{0}^{*}-d_{2}}\right),
$$

From (28) we have

$$
\frac{\partial \Phi_{1}\left(t, \zeta_{0}\right)}{\partial y_{1}}=-\lambda x_{0}^{*} e^{-\beta_{0} x_{0}^{*} t}\left(\frac{e^{\left(\beta_{0} x_{0}^{*}-d_{2}\right) t}-1}{\beta_{0} x_{0}^{*}-d_{2}}\right) .
$$




\subsection{Second derivatives of $\Phi_{3}$}

The second partial derivatives of $\Phi_{3}$ can be obtained from the following differential equations,

$\frac{d}{d t}\left(\frac{\partial^{2} \Phi_{3}\left(t, \zeta_{0}\right)}{\partial x_{0}^{2}}\right)=\frac{\partial F_{3}(\zeta(t))}{\partial x_{0}} \frac{\partial^{2} \Phi_{1}\left(t, \zeta_{0}\right)}{\partial x_{0}^{2}}+\frac{\partial F_{3}(\zeta(t))}{\partial x_{1}} \frac{\partial^{2} \Phi_{2}\left(t, \zeta_{0}\right)}{\partial x_{0}^{2}}+\frac{\partial F_{3}(\zeta(t))}{\partial y_{0}} \frac{\partial^{2} \Phi_{3}\left(t, \zeta_{0}\right)}{\partial x_{0}^{2}}+\frac{\partial F_{3}(\zeta(t))}{\partial y_{1}} \frac{\partial^{2} \Phi_{4}\left(t, \zeta_{0}\right)}{\partial x_{0}^{2}}$ $+\left(\frac{\partial^{2} F_{3}(\zeta(t))}{\partial x_{0}^{2}} \frac{\partial \Phi_{1}\left(t, \zeta_{0}\right)}{\partial x_{0}}+\frac{\partial^{2} F_{3}(\zeta(t))}{\partial x_{0} \partial x_{1}} \frac{\partial \Phi_{2}\left(t, \zeta_{0}\right)}{\partial x_{0}}+\frac{\partial^{2} F_{3}(\zeta(t))}{\partial x_{0} \partial y_{0}} \frac{\partial \Phi_{3}\left(t, \zeta_{0}\right)}{\partial x_{0}}+\frac{\partial^{2} F_{3}(\zeta(t))}{\partial x_{0} \partial y_{1}} \frac{\partial \Phi_{4}\left(t, \zeta_{0}\right)}{\partial x_{0}}\right) \frac{\partial \Phi_{1}\left(t, \zeta_{0}\right)}{\partial x_{0}}$

$+\left(\frac{\partial^{2} F_{3}(\zeta(t))}{\partial x_{0} \partial x_{1}} \frac{\partial \Phi_{1}\left(t, \zeta_{0}\right)}{\partial x_{0}}+\frac{\partial^{2} F_{3}(\zeta(t))}{\partial x_{1}^{2}} \frac{\partial \Phi_{2}\left(t, \zeta_{0}\right)}{\partial x_{0}}+\frac{\partial^{2} F_{3}(\zeta(t))}{\partial x_{1} \partial y_{0}} \frac{\partial \Phi_{3}\left(t, \zeta_{0}\right)}{\partial x_{0}}+\frac{\partial^{2} F_{3}(\zeta(t))}{\partial x_{1} \partial y_{1}} \frac{\partial \Phi_{4}\left(t, \zeta_{0}\right)}{\partial x_{0}}\right) \frac{\partial \Phi_{2}\left(t, \zeta_{0}\right)}{\partial x_{0}}$

$+\left(\frac{\partial^{2} F_{3}(\zeta(t))}{\partial x_{0} \partial y_{0}} \frac{\partial \Phi_{1}\left(t, \zeta_{0}\right)}{\partial x_{0}}+\frac{\partial^{2} F_{3}(\zeta(t))}{\partial x_{1} \partial y_{0}} \frac{\partial \Phi_{2}\left(t, \zeta_{0}\right)}{\partial x_{0}}+\frac{\partial^{2} F_{3}(\zeta(t))}{\partial y_{0}^{2}} \frac{\partial \Phi_{3}\left(t, \zeta_{0}\right)}{\partial x_{0}}+\frac{\partial^{2} F_{3}(\zeta(t))}{\partial y_{0} \partial y_{1}} \frac{\partial \Phi_{4}\left(t, \zeta_{0}\right)}{\partial x_{0}}\right) \frac{\partial \Phi_{3}\left(t, \zeta_{0}\right)}{\partial x_{0}}$

$+\left(\frac{\partial^{2} F_{3}(\zeta(t))}{\partial x_{0} \partial y_{1}} \frac{\partial \Phi_{1}\left(t, \zeta_{0}\right)}{\partial x_{0}}+\frac{\partial^{2} F_{3}(\zeta(t))}{\partial x_{1} \partial y_{1}} \frac{\partial \Phi_{2}\left(t, \zeta_{0}\right)}{\partial x_{0}}+\frac{\partial^{2} F_{3}(\zeta(t))}{\partial y_{0} \partial y_{1}} \frac{\partial \Phi_{3}\left(t, \zeta_{0}\right)}{\partial x_{0}}+\frac{\partial^{2} F_{3}(\zeta(t))}{\partial y_{1}^{2}} \frac{\partial \Phi_{4}\left(t, \zeta_{0}\right)}{\partial x_{0}}\right) \frac{\partial \Phi_{4}\left(t, \zeta_{0}\right)}{\partial x_{0}}$

with the initial condition $\frac{\partial^{2} \Phi_{3}\left(0, \zeta_{0}\right)}{\partial x_{0}^{2}}=0$, then

$$
\frac{d}{d t}\left(\frac{\partial^{2} \Phi_{3}\left(t, \zeta_{0}\right)}{\partial x_{0}^{2}}\right)=\frac{\partial F_{3}(\zeta(t))}{\partial y_{0}} \frac{\partial^{2} \Phi_{3}\left(t, \zeta_{0}\right)}{\partial x_{0}^{2}}
$$

with the initial condition $\frac{\partial^{2} \Phi_{3}\left(0, \zeta_{0}\right)}{\partial x_{0}^{2}}=0$. From (34) we have

$$
\frac{\partial^{2} \Phi_{3}\left(t, \zeta_{0}\right)}{\partial x_{0}^{2}}=0
$$

$\frac{d}{d t}\left(\frac{\partial^{2} \Phi_{3}\left(t, \zeta_{0}\right)}{\partial x_{0} \partial x_{1}}\right)=\frac{\partial F_{3}(\zeta(t))}{\partial x_{0}} \frac{\partial^{2} \Phi_{1}\left(t, \zeta_{0}\right)}{\partial x_{0} \partial x_{1}}+\frac{\partial F_{3}(\zeta(t))}{\partial x_{1}} \frac{\partial^{2} \Phi_{2}\left(t, \zeta_{0}\right)}{\partial x_{0} \partial x_{1}}+\frac{\partial F_{3}(\zeta(t))}{\partial y_{0}} \frac{\partial^{2} \Phi_{3}\left(t, \zeta_{0}\right)}{\partial x_{0} \partial x_{1}}+\frac{\partial F_{3}(\zeta(t))}{\partial y_{1}} \frac{\partial^{2} \Phi_{4}\left(t, \zeta_{0}\right)}{\partial x_{0} \partial x_{1}}$

$+\left(\frac{\partial^{2} F_{3}(\zeta(t))}{\partial x_{0}^{2}} \frac{\partial \Phi_{1}\left(t, \zeta_{0}\right)}{\partial x_{1}}+\frac{\partial^{2} F_{3}(\zeta(t))}{\partial x_{0} \partial x_{1}} \frac{\partial \Phi_{2}\left(t, \zeta_{0}\right)}{\partial x_{1}}+\frac{\partial^{2} F_{3}(\zeta(t))}{\partial x_{0} \partial y_{0}} \frac{\partial \Phi_{3}\left(t, \zeta_{0}\right)}{\partial x_{1}}+\frac{\partial^{2} F_{3}(\zeta(t))}{\partial x_{0} \partial y_{1}} \frac{\partial \Phi_{4}\left(t, \zeta_{0}\right)}{\partial x_{1}}\right) \frac{\partial \Phi_{1}\left(t, \zeta_{0}\right)}{\partial x_{0}}$

$+\left(\frac{\partial^{2} F_{3}(\zeta(t))}{\partial x_{0} \partial x_{1}} \frac{\partial \Phi_{1}\left(t, \zeta_{0}\right)}{\partial x_{1}}+\frac{\partial^{2} F_{3}(\zeta(t))}{\partial x_{1}^{2}} \frac{\partial \Phi_{2}\left(t, \zeta_{0}\right)}{\partial x_{1}}+\frac{\partial^{2} F_{3}(\zeta(t))}{\partial x_{1} \partial y_{0}} \frac{\partial \Phi_{3}\left(t, \zeta_{0}\right)}{\partial x_{1}}+\frac{\partial^{2} F_{3}(\zeta(t))}{\partial x_{1} \partial y_{1}} \frac{\partial \Phi_{4}\left(t, \zeta_{0}\right)}{\partial x_{1}}\right) \frac{\partial \Phi_{2}\left(t, \zeta_{0}\right)}{\partial x_{0}}$

$+\left(\frac{\partial^{2} F_{3}(\zeta(t))}{\partial x_{0} \partial y_{0}} \frac{\partial \Phi_{1}\left(t, \zeta_{0}\right)}{\partial x_{1}}+\frac{\partial^{2} F_{3}(\zeta(t))}{\partial x_{1} \partial y_{0}} \frac{\partial \Phi_{2}\left(t, \zeta_{0}\right)}{\partial x_{1}}+\frac{\partial^{2} F_{3}(\zeta(t))}{\partial y_{0}^{2}} \frac{\partial \Phi_{3}\left(t, \zeta_{0}\right)}{\partial x_{1}}+\frac{\partial^{2} F_{3}(\zeta(t))}{\partial y_{0} \partial y_{1}} \frac{\partial \Phi_{4}\left(t, \zeta_{0}\right)}{\partial x_{1}}\right) \frac{\partial \Phi_{3}\left(t, \zeta_{0}\right)}{\partial x_{0}}$

$+\left(\frac{\partial^{2} F_{3}(\zeta(t))}{\partial x_{0} \partial y_{1}} \frac{\partial \Phi_{1}\left(t, \zeta_{0}\right)}{\partial x_{1}}+\frac{\partial^{2} F_{3}(\zeta(t))}{\partial x_{1} \partial y_{1}} \frac{\partial \Phi_{2}\left(t, \zeta_{0}\right)}{\partial x_{1}}+\frac{\partial^{2} F_{3}(\zeta(t))}{\partial y_{0} \partial y_{1}} \frac{\partial \Phi_{3}\left(t, \zeta_{0}\right)}{\partial x_{1}}+\frac{\partial^{2} F_{3}(\zeta(t))}{\partial y_{1}^{2}} \frac{\partial \Phi_{4}\left(t, \zeta_{0}\right)}{\partial x_{1}}\right) \frac{\partial \Phi_{4}\left(t, \zeta_{0}\right)}{\partial x_{0}}$

with the initial condition $\frac{\partial^{2} \Phi_{3}\left(0, \zeta_{0}\right)}{\partial x_{0} \partial x_{1}}=0$, then

$$
\frac{d}{d t}\left(\frac{\partial^{2} \Phi_{3}\left(t, \zeta_{0}\right)}{\partial x_{0} \partial x_{1}}\right)=\frac{\partial F_{3}(\zeta(t))}{\partial y_{0}} \frac{\partial^{2} \Phi_{3}\left(t, \zeta_{0}\right)}{\partial x_{0} \partial x_{1}}
$$

with the initial condition $\frac{\partial^{2} \Phi_{3}\left(0, \zeta_{0}\right)}{\partial x_{0} \partial x_{1}}=0$. From (35) we have

$$
\frac{\partial^{2} \Phi_{3}\left(t, \zeta_{0}\right)}{\partial x_{0} \partial x_{1}}=0
$$

$\frac{d}{d t}\left(\frac{\partial^{2} \Phi_{3}\left(t, \zeta_{0}\right)}{\partial x_{0} \partial y_{0}}\right)=\frac{\partial F_{3}(\zeta(t))}{\partial x_{0}} \frac{\partial^{2} \Phi_{1}\left(t, \zeta_{0}\right)}{\partial x_{0} \partial y_{0}}+\frac{\partial F_{3}(\zeta(t))}{\partial x_{1}} \frac{\partial^{2} \Phi_{2}\left(t, \zeta_{0}\right)}{\partial x_{0} \partial y_{0}}+\frac{\partial F_{3}(\zeta(t))}{\partial y_{0}} \frac{\partial^{2} \Phi_{3}\left(t, \zeta_{0}\right)}{\partial x_{0} \partial y_{0}}+\frac{\partial F_{3}(\zeta(t))}{\partial y_{1}} \frac{\partial^{2} \Phi_{4}\left(t, \zeta_{0}\right)}{\partial x_{0} \partial y_{0}}$

$+\left(\frac{\partial^{2} F_{3}(\zeta(t))}{\partial x_{0}^{2}} \frac{\partial \Phi_{1}\left(t, \zeta_{0}\right)}{\partial y_{0}}+\frac{\partial^{2} F_{3}(\zeta(t))}{\partial x_{0} \partial x_{1}} \frac{\partial \Phi_{2}\left(t, \zeta_{0}\right)}{\partial y_{0}}+\frac{\partial^{2} F_{3}(\zeta(t))}{\partial x_{0} \partial y_{0}} \frac{\partial \Phi_{3}\left(t, \zeta_{0}\right)}{\partial y_{0}}+\frac{\partial^{2} F_{3}(\zeta(t))}{\partial x_{0} \partial y_{1}} \frac{\partial \Phi_{4}\left(t, \zeta_{0}\right)}{\partial y_{0}}\right) \frac{\partial \Phi_{1}\left(t, \zeta_{0}\right)}{\partial x_{0}}$

$+\left(\frac{\partial^{2} F_{3}(\zeta(t))}{\partial x_{0} \partial x_{1}} \frac{\partial \Phi_{1}\left(t, \zeta_{0}\right)}{\partial y_{0}}+\frac{\partial^{2} F_{3}(\zeta(t))}{\partial x_{1}^{2}} \frac{\partial \Phi_{2}\left(t, \zeta_{0}\right)}{\partial y_{0}}+\frac{\partial^{2} F_{3}(\zeta(t))}{\partial x_{1} \partial y_{0}} \frac{\partial \Phi_{3}\left(t, \zeta_{0}\right)}{\partial y_{0}}+\frac{\partial^{2} F_{3}(\zeta(t))}{\partial x_{1} \partial y_{1}} \frac{\partial \Phi_{4}\left(t, \zeta_{0}\right)}{\partial y_{0}}\right) \frac{\partial \Phi_{2}\left(t, \zeta_{0}\right)}{\partial x_{0}}$

$+\left(\frac{\partial^{2} F_{3}(\zeta(t))}{\partial x_{0} \partial y_{0}} \frac{\partial \Phi_{1}\left(t, \zeta_{0}\right)}{\partial y_{0}}+\frac{\partial^{2} F_{3}(\zeta(t))}{\partial x_{1} \partial y_{0}} \frac{\partial \Phi_{2}\left(t, \zeta_{0}\right)}{\partial y_{0}}+\frac{\partial^{2} F_{3}(\zeta(t))}{\partial y_{0}^{2}} \frac{\partial \Phi_{3}\left(t, \zeta_{0}\right)}{\partial y_{0}}+\frac{\partial^{2} F_{3}(\zeta(t))}{\partial y_{0} \partial y_{1}} \frac{\partial \Phi_{4}\left(t, \zeta_{0}\right)}{\partial y_{0}}\right) \frac{\partial \Phi_{3}\left(t, \zeta_{0}\right)}{\partial x_{0}}$

$+\left(\frac{\partial^{2} F_{3}(\zeta(t))}{\partial x_{0} \partial y_{1}} \frac{\partial \Phi_{1}\left(t, \zeta_{0}\right)}{\partial y_{0}}+\frac{\partial^{2} F_{3}(\zeta(t))}{\partial x_{1} \partial y_{1}} \frac{\partial \Phi_{2}\left(t, \zeta_{0}\right)}{\partial y_{0}}+\frac{\partial^{2} F_{3}(\zeta(t))}{\partial y_{0} \partial y_{1}} \frac{\partial \Phi_{3}\left(t, \zeta_{0}\right)}{\partial y_{0}}+\frac{\partial^{2} F_{3}(\zeta(t))}{\partial y_{1}^{2}} \frac{\partial \Phi_{4}\left(t, \zeta_{0}\right)}{\partial y_{0}}\right) \frac{\partial \Phi_{4}\left(t, \zeta_{0}\right)}{\partial x_{0}}$ 
with the initial condition $\frac{\partial^{2} \Phi_{3}\left(0, \zeta_{0}\right)}{\partial x_{0} \partial y_{0}}=0$, then

$$
\frac{d}{d t}\left(\frac{\partial^{2} \Phi_{3}\left(t, \zeta_{0}\right)}{\partial x_{0} \partial y_{0}}\right)=\frac{\partial F_{3}(\zeta(t))}{\partial y_{0}} \frac{\partial^{2} \Phi_{3}\left(t, \zeta_{0}\right)}{\partial x_{0} \partial y_{0}}+\frac{\partial^{2} F_{3}(\zeta(t))}{\partial x_{1} \partial y_{0}} \frac{\partial \Phi_{3}\left(t, \zeta_{0}\right)}{\partial y_{0}} \frac{\partial \Phi_{2}\left(t, \zeta_{0}\right)}{\partial x_{0}}
$$

with the initial condition $\frac{\partial^{2} \Phi_{3}\left(0, \zeta_{0}\right)}{\partial x_{0} \partial y_{0}}=0$. From (36) we have

$$
\frac{\partial^{2} \Phi_{3}\left(t, \zeta_{0}\right)}{\partial x_{0} \partial y_{0}}=e^{\frac{\partial F_{3}(\zeta(t))}{\partial y_{0}} t} \int_{0}^{t} \frac{\partial^{2} F_{3}(\zeta(s))}{\partial x_{1} \partial y_{0}} \frac{\partial \Phi_{2}\left(s, \zeta_{0}\right)}{\partial x_{0}} d s .
$$

$\frac{d}{d t}\left(\frac{\partial^{2} \Phi_{3}\left(t, \zeta_{0}\right)}{\partial x_{0} \partial y_{1}}\right)=\frac{\partial F_{3}(\zeta(t))}{\partial x_{0}} \frac{\partial^{2} \Phi_{1}\left(t, \zeta_{0}\right)}{\partial x_{0} \partial y_{1}}+\frac{\partial F_{3}(\zeta(t))}{\partial x_{1}} \frac{\partial^{2} \Phi_{2}\left(t, \zeta_{0}\right)}{\partial x_{0} \partial y_{1}}+\frac{\partial F_{3}(\zeta(t))}{\partial y_{0}} \frac{\partial^{2} \Phi_{3}\left(t, \zeta_{0}\right)}{\partial x_{0} \partial y_{1}}+\frac{\partial F_{3}(\zeta(t))}{\partial y_{1}} \frac{\partial^{2} \Phi_{4}\left(t, \zeta_{0}\right)}{\partial x_{0} \partial y_{1}}$

$+\left(\frac{\partial^{2} F_{3}(\zeta(t))}{\partial x_{0}^{2}} \frac{\partial \Phi_{1}\left(t, \zeta_{0}\right)}{\partial y_{1}}+\frac{\partial^{2} F_{3}(\zeta(t))}{\partial x_{0} \partial x_{1}} \frac{\partial \Phi_{2}\left(t, \zeta_{0}\right)}{\partial y_{1}}+\frac{\partial^{2} F_{3}(\zeta(t))}{\partial x_{0} \partial y_{0}} \frac{\partial \Phi_{3}\left(t, \zeta_{0}\right)}{\partial y_{1}}+\frac{\partial^{2} F_{3}(\zeta(t))}{\partial x_{0} \partial y_{1}} \frac{\partial \Phi_{4}\left(t, \zeta_{0}\right)}{\partial y_{1}}\right) \frac{\partial \Phi_{1}\left(t, \zeta_{0}\right)}{\partial x_{0}}$

$+\left(\frac{\partial^{2} F_{3}(\zeta(t))}{\partial x_{0} \partial x_{1}} \frac{\partial \Phi_{1}\left(t, \zeta_{0}\right)}{\partial y_{1}}+\frac{\partial^{2} F_{3}(\zeta(t))}{\partial x_{1}^{2}} \frac{\partial \Phi_{2}\left(t, \zeta_{0}\right)}{\partial y_{1}}+\frac{\partial^{2} F_{3}(\zeta(t))}{\partial x_{1} \partial y_{0}} \frac{\partial \Phi_{3}\left(t, \zeta_{0}\right)}{\partial y_{1}}+\frac{\partial^{2} F_{3}(\zeta(t))}{\partial x_{1} \partial y_{1}} \frac{\partial \Phi_{4}\left(t, \zeta_{0}\right)}{\partial y_{1}}\right) \frac{\partial \Phi_{2}\left(t, \zeta_{0}\right)}{\partial x_{0}}$

$+\left(\frac{\partial^{2} F_{3}(\zeta(t))}{\partial x_{0} \partial y_{0}} \frac{\partial \Phi_{1}\left(t, \zeta_{0}\right)}{\partial y_{1}}+\frac{\partial^{2} F_{3}(\zeta(t))}{\partial x_{1} \partial y_{0}} \frac{\partial \Phi_{2}\left(t, \zeta_{0}\right)}{\partial y_{1}}+\frac{\partial^{2} F_{3}(\zeta(t))}{\partial y_{0}^{2}} \frac{\partial \Phi_{3}\left(t, \zeta_{0}\right)}{\partial y_{1}}+\frac{\partial^{2} F_{3}(\zeta(t))}{\partial y_{0} \partial y_{1}} \frac{\partial \Phi_{4}\left(t, \zeta_{0}\right)}{\partial y_{1}}\right) \frac{\partial \Phi_{3}\left(t, \zeta_{0}\right)}{\partial x_{0}}$

$+\left(\frac{\partial^{2} F_{3}(\zeta(t))}{\partial x_{0} \partial y_{1}} \frac{\partial \Phi_{1}\left(t, \zeta_{0}\right)}{\partial y_{1}}+\frac{\partial^{2} F_{3}(\zeta(t))}{\partial x_{1} \partial y_{1}} \frac{\partial \Phi_{2}\left(t, \zeta_{0}\right)}{\partial y_{1}}+\frac{\partial^{2} F_{3}(\zeta(t))}{\partial y_{0} \partial y_{1}} \frac{\partial \Phi_{3}\left(t, \zeta_{0}\right)}{\partial y_{1}}+\frac{\partial^{2} F_{3}(\zeta(t))}{\partial y_{1}^{2}} \frac{\partial \Phi_{4}\left(t, \zeta_{0}\right)}{\partial y_{1}}\right) \frac{\partial \Phi_{4}\left(t, \zeta_{0}\right)}{\partial x_{0}}$

with the initial condition $\frac{\partial^{2} \Phi_{3}\left(0, \zeta_{0}\right)}{\partial x_{0} \partial y_{1}}=0$, then

$$
\frac{d}{d t}\left(\frac{\partial^{2} \Phi_{3}\left(t, \zeta_{0}\right)}{\partial x_{0} \partial y_{1}}\right)=\frac{\partial F_{3}(\zeta(t))}{\partial y_{0}} \frac{\partial^{2} \Phi_{3}\left(t, \zeta_{0}\right)}{\partial x_{0} \partial y_{1}}
$$

with the initial condition $\frac{\partial^{2} \Phi_{3}\left(0, \zeta_{0}\right)}{\partial x_{0} \partial y_{1}}=0$. From (37) we have

$$
\frac{\partial^{2} \Phi_{3}\left(t, \zeta_{0}\right)}{\partial x_{0} \partial y_{1}}=0
$$

$\frac{d}{d t}\left(\frac{\partial^{2} \Phi_{3}\left(t, \zeta_{0}\right)}{\partial x_{1}^{2}}\right)=\frac{\partial F_{3}(\zeta(t))}{\partial x_{0}} \frac{\partial^{2} \Phi_{1}\left(t, \zeta_{0}\right)}{\partial x_{1}^{2}}+\frac{\partial F_{3}(\zeta(t))}{\partial x_{1}} \frac{\partial^{2} \Phi_{2}\left(t, \zeta_{0}\right)}{\partial x_{1}^{2}}+\frac{\partial F_{3}(\zeta(t))}{\partial y_{0}} \frac{\partial^{2} \Phi_{3}\left(t, \zeta_{0}\right)}{\partial x_{1}^{2}}+\frac{\partial F_{3}(\zeta(t))}{\partial y_{1}} \frac{\partial^{2} \Phi_{4}\left(t, \zeta_{0}\right)}{\partial x_{1}^{2}}$ $+\left(\frac{\partial^{2} F_{3}(\zeta(t))}{\partial x_{0}^{2}} \frac{\partial \Phi_{1}\left(t, \zeta_{0}\right)}{\partial x_{1}}+\frac{\partial^{2} F_{3}(\zeta(t))}{\partial x_{0} \partial x_{1}} \frac{\partial \Phi_{2}\left(t, \zeta_{0}\right)}{\partial x_{1}}+\frac{\partial^{2} F_{3}(\zeta(t))}{\partial x_{0} \partial y_{0}} \frac{\partial \Phi_{3}\left(t, \zeta_{0}\right)}{\partial x_{1}}+\frac{\partial^{2} F_{3}(\zeta(t))}{\partial x_{0} \partial y_{1}} \frac{\partial \Phi_{4}\left(t, \zeta_{0}\right)}{\partial x_{1}}\right) \frac{\partial \Phi_{1}\left(t, \zeta_{0}\right)}{\partial x_{1}}$ $+\left(\frac{\partial^{2} F_{3}(\zeta(t))}{\partial x_{0} \partial x_{1}} \frac{\partial \Phi_{1}\left(t, \zeta_{0}\right)}{\partial x_{1}}+\frac{\partial^{2} F_{3}(\zeta(t))}{\partial x_{1}^{2}} \frac{\partial \Phi_{2}\left(t, \zeta_{0}\right)}{\partial x_{1}}+\frac{\partial^{2} F_{3}(\zeta(t))}{\partial x_{1} \partial y_{0}} \frac{\partial \Phi_{3}\left(t, \zeta_{0}\right)}{\partial x_{1}}+\frac{\partial^{2} F_{3}(\zeta(t))}{\partial x_{1} \partial y_{1}} \frac{\partial \Phi_{4}\left(t, \zeta_{0}\right)}{\partial x_{1}}\right) \frac{\partial \Phi_{2}\left(t, \zeta_{0}\right)}{\partial x_{1}}$ $+\left(\frac{\partial^{2} F_{3}(\zeta(t))}{\partial x_{0} \partial y_{0}} \frac{\partial \Phi_{1}\left(t, \zeta_{0}\right)}{\partial x_{1}}+\frac{\partial^{2} F_{3}(\zeta(t))}{\partial x_{1} \partial y_{0}} \frac{\partial \Phi_{2}\left(t, \zeta_{0}\right)}{\partial x_{1}}+\frac{\partial^{2} F_{3}(\zeta(t))}{\partial y_{0}^{2}} \frac{\partial \Phi_{3}\left(t, \zeta_{0}\right)}{\partial x_{1}}+\frac{\partial^{2} F_{3}(\zeta(t))}{\partial y_{0} \partial y_{1}} \frac{\partial \Phi_{4}\left(t, \zeta_{0}\right)}{\partial x_{1}}\right) \frac{\partial \Phi_{3}\left(t, \zeta_{0}\right)}{\partial x_{1}}$ $+\left(\frac{\partial^{2} F_{3}(\zeta(t))}{\partial x_{0} \partial y_{1}} \frac{\partial \Phi_{1}\left(t, \zeta_{0}\right)}{\partial x_{1}}+\frac{\partial^{2} F_{3}(\zeta(t))}{\partial x_{1} \partial y_{1}} \frac{\partial \Phi_{2}\left(t, \zeta_{0}\right)}{\partial x_{1}}+\frac{\partial^{2} F_{3}(\zeta(t))}{\partial y_{0} \partial y_{1}} \frac{\partial \Phi_{3}\left(t, \zeta_{0}\right)}{\partial x_{1}}+\frac{\partial^{2} F_{3}(\zeta(t))}{\partial y_{1}^{2}} \frac{\partial \Phi_{4}\left(t, \zeta_{0}\right)}{\partial x_{1}}\right) \frac{\partial \Phi_{4}\left(t, \zeta_{0}\right)}{\partial x_{1}}$ with the initial condition $\frac{\partial^{2} \Phi_{3}\left(0, \zeta_{0}\right)}{\partial x_{1}^{2}}=0$, then

$$
\frac{d}{d t}\left(\frac{\partial^{2} \Phi_{3}\left(t, \zeta_{0}\right)}{\partial x_{1}^{2}}\right)=\frac{\partial F_{3}(\zeta(t))}{\partial y_{0}} \frac{\partial^{2} \Phi_{3}\left(t, \zeta_{0}\right)}{\partial x_{1}^{2}}
$$

with the initial condition $\frac{\partial^{2} \Phi_{3}\left(0, \zeta_{0}\right)}{\partial x_{1}^{2}}=0$. From (38) we have

$$
\frac{\partial^{2} \Phi_{3}\left(t, \zeta_{0}\right)}{\partial x_{1}^{2}}=0
$$

$\frac{d}{d t}\left(\frac{\partial^{2} \Phi_{3}\left(t, \zeta_{0}\right)}{\partial x_{1} \partial y_{0}}\right)=\frac{\partial F_{3}(\zeta(t))}{\partial x_{0}} \frac{\partial^{2} \Phi_{1}\left(t, \zeta_{0}\right)}{\partial x_{1} \partial y_{0}}+\frac{\partial F_{3}(\zeta(t))}{\partial x_{1}} \frac{\partial^{2} \Phi_{2}\left(t, \zeta_{0}\right)}{\partial x_{1} \partial y_{0}}+\frac{\partial F_{3}(\zeta(t))}{\partial y_{0}} \frac{\partial^{2} \Phi_{3}\left(t, \zeta_{0}\right)}{\partial x_{1} \partial y_{0}}+\frac{\partial F_{3}(\zeta(t))}{\partial y_{1}} \frac{\partial^{2} \Phi_{4}\left(t, \zeta_{0}\right)}{\partial x_{1} \partial y_{0}}$ $+\left(\frac{\partial^{2} F_{3}(\zeta(t))}{\partial x_{0}^{2}} \frac{\partial \Phi_{1}\left(t, \zeta_{0}\right)}{\partial y_{0}}+\frac{\partial^{2} F_{3}(\zeta(t))}{\partial x_{0} \partial x_{1}} \frac{\partial \Phi_{2}\left(t, \zeta_{0}\right)}{\partial y_{0}}+\frac{\partial^{2} F_{3}(\zeta(t))}{\partial x_{0} \partial y_{0}} \frac{\partial \Phi_{3}\left(t, \zeta_{0}\right)}{\partial y_{0}}+\frac{\partial^{2} F_{3}(\zeta(t))}{\partial x_{0} \partial y_{1}} \frac{\partial \Phi_{4}\left(t, \zeta_{0}\right)}{\partial y_{0}}\right) \frac{\partial \Phi_{1}\left(t, \zeta_{0}\right)}{\partial x_{1}}$ $+\left(\frac{\partial^{2} F_{3}(\zeta(t))}{\partial x_{0} \partial x_{1}} \frac{\partial \Phi_{1}\left(t, \zeta_{0}\right)}{\partial y_{0}}+\frac{\partial^{2} F_{3}(\zeta(t))}{\partial x_{1}^{2}} \frac{\partial \Phi_{2}\left(t, \zeta_{0}\right)}{\partial y_{0}}+\frac{\partial^{2} F_{3}(\zeta(t))}{\partial x_{1} \partial y_{0}} \frac{\partial \Phi_{3}\left(t, \zeta_{0}\right)}{\partial y_{0}}+\frac{\partial^{2} F_{3}(\zeta(t))}{\partial x_{1} \partial y_{1}} \frac{\partial \Phi_{4}\left(t, \zeta_{0}\right)}{\partial y_{0}}\right) \frac{\partial \Phi_{2}\left(t, \zeta_{0}\right)}{\partial x_{1}}$ $+\left(\frac{\partial^{2} F_{3}(\zeta(t))}{\partial x_{0} \partial y_{0}} \frac{\partial \Phi_{1}\left(t, \zeta_{0}\right)}{\partial y_{0}}+\frac{\partial^{2} F_{3}(\zeta(t))}{\partial x_{1} \partial y_{0}} \frac{\partial \Phi_{2}\left(t, \zeta_{0}\right)}{\partial y_{0}}+\frac{\partial^{2} F_{3}(\zeta(t))}{\partial y_{0}^{2}} \frac{\partial \Phi_{3}\left(t, \zeta_{0}\right)}{\partial y_{0}}+\frac{\partial^{2} F_{3}(\zeta(t))}{\partial y_{0} \partial y_{1}} \frac{\partial \Phi_{4}\left(t, \zeta_{0}\right)}{\partial y_{0}}\right) \frac{\partial \Phi_{3}\left(t, \zeta_{0}\right)}{\partial x_{1}}$ $+\left(\frac{\partial^{2} F_{3}(\zeta(t))}{\partial x_{0} \partial y_{1}} \frac{\partial \Phi_{1}\left(t, \zeta_{0}\right)}{\partial y_{0}}+\frac{\partial^{2} F_{3}(\zeta(t))}{\partial x_{1} \partial y_{1}} \frac{\partial \Phi_{2}\left(t, \zeta_{0}\right)}{\partial y_{0}}+\frac{\partial^{2} F_{3}(\zeta(t))}{\partial y_{0} \partial y_{1}} \frac{\partial \Phi_{3}\left(t, \zeta_{0}\right)}{\partial y_{0}}+\frac{\partial^{2} F_{3}(\zeta(t))}{\partial y_{1}^{2}} \frac{\partial \Phi_{4}\left(t, \zeta_{0}\right)}{\partial y_{0}}\right) \frac{\partial \Phi_{4}\left(t, \zeta_{0}\right)}{\partial x_{1}}$ 
with the initial condition $\frac{\partial^{2} \Phi_{3}\left(0, \zeta_{0}\right)}{\partial x_{1} \partial y_{0}}=0$, then

$$
\frac{d}{d t}\left(\frac{\partial^{2} \Phi_{3}\left(t, \zeta_{0}\right)}{\partial x_{1} \partial y_{0}}\right)=\frac{\partial F_{3}(\zeta(t))}{\partial y_{0}} \frac{\partial^{2} \Phi_{3}\left(t, \zeta_{0}\right)}{\partial x_{1} \partial y_{0}}+\frac{\partial^{2} F_{3}(\zeta(t))}{\partial x_{1} \partial y_{0}} \frac{\partial \Phi_{3}\left(t, \zeta_{0}\right)}{\partial y_{0}} \frac{\partial \Phi_{2}\left(t, \zeta_{0}\right)}{\partial x_{1}}
$$

with the initial condition $\frac{\partial^{2} \Phi_{3}\left(0, \zeta_{0}\right)}{\partial x_{1} \partial y_{0}}=0$. From (39) we have

$$
\frac{\partial^{2} \Phi_{3}\left(t, \zeta_{0}\right)}{\partial x_{1} \partial y_{0}}=e^{\frac{\partial F_{3}(\zeta(t))}{\partial y_{0}} t} \int_{0}^{t} \frac{\partial^{2} F_{3}(\zeta(s))}{\partial x_{1} \partial y_{0}} \frac{\partial \Phi_{2}\left(s, \zeta_{0}\right)}{\partial x_{1}} d s
$$

$\frac{d}{d t}\left(\frac{\partial^{2} \Phi_{3}\left(t, \zeta_{0}\right)}{\partial x_{1} \partial y_{1}}\right)=\frac{\partial F_{3}(\zeta(t))}{\partial x_{0}} \frac{\partial^{2} \Phi_{1}\left(t, \zeta_{0}\right)}{\partial x_{1} \partial y_{1}}+\frac{\partial F_{3}(\zeta(t))}{\partial x_{1}} \frac{\partial^{2} \Phi_{2}\left(t, \zeta_{0}\right)}{\partial x_{1} \partial y_{1}}+\frac{\partial F_{3}(\zeta(t))}{\partial y_{0}} \frac{\partial^{2} \Phi_{3}\left(t, \zeta_{0}\right)}{\partial x_{1} \partial y_{1}}+\frac{\partial F_{3}(\zeta(t))}{\partial y_{1}} \frac{\partial^{2} \Phi_{4}\left(t, \zeta_{0}\right)}{\partial x_{1} \partial y_{1}}$

$+\left(\frac{\partial^{2} F_{3}(\zeta(t))}{\partial x_{0}^{2}} \frac{\partial \Phi_{1}\left(t, \zeta_{0}\right)}{\partial y_{1}}+\frac{\partial^{2} F_{3}(\zeta(t))}{\partial x_{0} \partial x_{1}} \frac{\partial \Phi_{2}\left(t, \zeta_{0}\right)}{\partial y_{1}}+\frac{\partial^{2} F_{3}(\zeta(t))}{\partial x_{0} \partial y_{0}} \frac{\partial \Phi_{3}\left(t, \zeta_{0}\right)}{\partial y_{1}}+\frac{\partial^{2} F_{3}(\zeta(t))}{\partial x_{0} \partial y_{1}} \frac{\partial \Phi_{4}\left(t, \zeta_{0}\right)}{\partial y_{1}}\right) \frac{\partial \Phi_{1}\left(t, \zeta_{0}\right)}{\partial x_{1}}$

$+\left(\frac{\partial^{2} F_{3}(\zeta(t))}{\partial x_{0} \partial x_{1}} \frac{\partial \Phi_{1}\left(t, \zeta_{0}\right)}{\partial y_{1}}+\frac{\partial^{2} F_{3}(\zeta(t))}{\partial x_{1}^{2}} \frac{\partial \Phi_{2}\left(t, \zeta_{0}\right)}{\partial y_{1}}+\frac{\partial^{2} F_{3}(\zeta(t))}{\partial x_{1} \partial y_{0}} \frac{\partial \Phi_{3}\left(t, \zeta_{0}\right)}{\partial y_{1}}+\frac{\partial^{2} F_{3}(\zeta(t))}{\partial x_{1} \partial y_{1}} \frac{\partial \Phi_{4}\left(t, \zeta_{0}\right)}{\partial y_{1}}\right) \frac{\partial \Phi_{2}\left(t, \zeta_{0}\right)}{\partial x_{1}}$

$+\left(\frac{\partial^{2} F_{3}(\zeta(t))}{\partial x_{0} \partial y_{0}} \frac{\partial \Phi_{1}\left(t, \zeta_{0}\right)}{\partial y_{1}}+\frac{\partial^{2} F_{3}(\zeta(t))}{\partial x_{1} \partial y_{0}} \frac{\partial \Phi_{2}\left(t, \zeta_{0}\right)}{\partial y_{1}}+\frac{\partial^{2} F_{3}(\zeta(t))}{\partial y_{0}^{2}} \frac{\partial \Phi_{3}\left(t, \zeta_{0}\right)}{\partial y_{1}}+\frac{\partial^{2} F_{3}(\zeta(t))}{\partial y_{0} \partial y_{1}} \frac{\partial \Phi_{4}\left(t, \zeta_{0}\right)}{\partial y_{1}}\right) \frac{\partial \Phi_{3}\left(t, \zeta_{0}\right)}{\partial x_{1}}$

$+\left(\frac{\partial^{2} F_{3}(\zeta(t))}{\partial x_{0} \partial y_{1}} \frac{\partial \Phi_{1}\left(t, \zeta_{0}\right)}{\partial y_{1}}+\frac{\partial^{2} F_{3}(\zeta(t))}{\partial x_{1} \partial y_{1}} \frac{\partial \Phi_{2}\left(t, \zeta_{0}\right)}{\partial y_{1}}+\frac{\partial^{2} F_{3}(\zeta(t))}{\partial y_{0} \partial y_{1}} \frac{\partial \Phi_{3}\left(t, \zeta_{0}\right)}{\partial y_{1}}+\frac{\partial^{2} F_{3}(\zeta(t))}{\partial y_{1}^{2}} \frac{\partial \Phi_{4}\left(t, \zeta_{0}\right)}{\partial y_{1}}\right) \frac{\partial \Phi_{4}\left(t, \zeta_{0}\right)}{\partial x_{1}}$

with the initial condition $\frac{\partial^{2} \Phi_{3}\left(0, \zeta_{0}\right)}{\partial x_{1} \partial y_{1}}=0$, then

$$
\frac{d}{d t}\left(\frac{\partial^{2} \Phi_{3}\left(t, \zeta_{0}\right)}{\partial x_{1} \partial y_{1}}\right)=\frac{\partial F_{3}(\zeta(t))}{\partial y_{0}} \frac{\partial^{2} \Phi_{3}\left(t, \zeta_{0}\right)}{\partial x_{1} \partial y_{1}}
$$

with the initial condition $\frac{\partial^{2} \Phi_{3}\left(0, \zeta_{0}\right)}{\partial x_{1} \partial y_{1}}=0$. From (40) we have

$$
\frac{\partial^{2} \Phi_{3}\left(t, \zeta_{0}\right)}{\partial x_{1} \partial y_{1}}=0
$$

$\frac{d}{d t}\left(\frac{\partial^{2} \Phi_{3}\left(t, \zeta_{0}\right)}{\partial y_{0}^{2}}\right)=\frac{\partial F_{3}(\zeta(t))}{\partial x_{0}} \frac{\partial^{2} \Phi_{1}\left(t, \zeta_{0}\right)}{\partial y_{0}^{2}}+\frac{\partial F_{3}(\zeta(t))}{\partial x_{1}} \frac{\partial^{2} \Phi_{2}\left(t, \zeta_{0}\right)}{\partial y_{0}^{2}}+\frac{\partial F_{3}(\zeta(t))}{\partial y_{0}} \frac{\partial^{2} \Phi_{3}\left(t, \zeta_{0}\right)}{\partial y_{0}^{2}}+\frac{\partial F_{3}(\zeta(t))}{\partial y_{1}} \frac{\partial^{2} \Phi_{4}\left(t, \zeta_{0}\right)}{\partial y_{0}^{2}}$ $+\left(\frac{\partial^{2} F_{3}(\zeta(t))}{\partial x_{0}^{2}} \frac{\partial \Phi_{1}\left(t, \zeta_{0}\right)}{\partial y_{0}}+\frac{\partial^{2} F_{3}(\zeta(t))}{\partial x_{0} \partial x_{1}} \frac{\partial \Phi_{2}\left(t, \zeta_{0}\right)}{\partial y_{0}}+\frac{\partial^{2} F_{3}(\zeta(t))}{\partial x_{0} \partial y_{0}} \frac{\partial \Phi_{3}\left(t, \zeta_{0}\right)}{\partial y_{0}}+\frac{\partial^{2} F_{3}(\zeta(t))}{\partial x_{0} \partial y_{1}} \frac{\partial \Phi_{4}\left(t, \zeta_{0}\right)}{\partial y_{0}}\right) \frac{\partial \Phi_{1}\left(t, \zeta_{0}\right)}{\partial y_{0}}$ $+\left(\frac{\partial^{2} F_{3}(\zeta(t))}{\partial x_{0} \partial x_{1}} \frac{\partial \Phi_{1}\left(t, \zeta_{0}\right)}{\partial y_{0}}+\frac{\partial^{2} F_{3}(\zeta(t))}{\partial x_{1}^{2}} \frac{\partial \Phi_{2}\left(t, \zeta_{0}\right)}{\partial y_{0}}+\frac{\partial^{2} F_{3}(\zeta(t))}{\partial x_{1} \partial y_{0}} \frac{\partial \Phi_{3}\left(t, \zeta_{0}\right)}{\partial y_{0}}+\frac{\partial^{2} F_{3}(\zeta(t))}{\partial x_{1} \partial y_{1}} \frac{\partial \Phi_{4}\left(t, \zeta_{0}\right)}{\partial y_{0}}\right) \frac{\partial \Phi_{2}\left(t, \zeta_{0}\right)}{\partial y_{0}}$ $+\left(\frac{\partial^{2} F_{3}(\zeta(t))}{\partial x_{0} \partial y_{0}} \frac{\partial \Phi_{1}\left(t, \zeta_{0}\right)}{\partial y_{0}}+\frac{\partial^{2} F_{3}(\zeta(t))}{\partial x_{1} \partial y_{0}} \frac{\partial \Phi_{2}\left(t, \zeta_{0}\right)}{\partial y_{0}}+\frac{\partial^{2} F_{3}(\zeta(t))}{\partial y_{0}^{2}} \frac{\partial \Phi_{3}\left(t, \zeta_{0}\right)}{\partial y_{0}}+\frac{\partial^{2} F_{3}(\zeta(t))}{\partial y_{0} \partial y_{1}} \frac{\partial \Phi_{4}\left(t, \zeta_{0}\right)}{\partial y_{0}}\right) \frac{\partial \Phi_{3}\left(t, \zeta_{0}\right)}{\partial y_{0}}$ $+\left(\frac{\partial^{2} F_{3}(\zeta(t))}{\partial x_{0} \partial y_{1}} \frac{\partial \Phi_{1}\left(t, \zeta_{0}\right)}{\partial y_{0}}+\frac{\partial^{2} F_{3}(\zeta(t))}{\partial x_{1} \partial y_{1}} \frac{\partial \Phi_{2}\left(t, \zeta_{0}\right)}{\partial y_{0}}+\frac{\partial^{2} F_{3}(\zeta(t))}{\partial y_{0} \partial y_{1}} \frac{\partial \Phi_{3}\left(t, \zeta_{0}\right)}{\partial y_{0}}+\frac{\partial^{2} F_{3}(\zeta(t))}{\partial y_{1}^{2}} \frac{\partial \Phi_{4}\left(t, \zeta_{0}\right)}{\partial y_{0}}\right) \frac{\partial \Phi_{4}\left(t, \zeta_{0}\right)}{\partial y_{0}}$ with the initial condition $\frac{\partial^{2} \Phi_{3}\left(0, \zeta_{0}\right)}{\partial y_{0}^{2}}=0$, then $\frac{d}{d t}\left(\frac{\partial^{2} \Phi_{3}\left(t, \zeta_{0}\right)}{\partial y_{0}^{2}}\right)=\frac{\partial F_{3}(\zeta(t))}{\partial y_{0}} \frac{\partial^{2} \Phi_{3}\left(t, \zeta_{0}\right)}{\partial y_{0}^{2}}+\left(\frac{\partial^{2} F_{3}(\zeta(t))}{\partial y_{0}^{2}} \frac{\partial \Phi_{3}\left(t, \zeta_{0}\right)}{\partial y_{0}}+\frac{\partial^{2} F_{3}(\zeta(t))}{\partial y_{0} \partial y_{1}} \frac{\partial \Phi_{4}\left(t, \zeta_{0}\right)}{\partial y_{0}}\right) \frac{\partial \Phi_{3}\left(t, \zeta_{0}\right)}{\partial y_{0}}$ with the initial condition $\frac{\partial^{2} \Phi_{3}\left(0, \zeta_{0}\right)}{\partial y_{0}^{2}}=0$. From (41) we have

$$
\begin{aligned}
& \frac{\partial^{2} \Phi_{3}\left(t, \zeta_{0}\right)}{\partial y_{0}^{2}}=e^{\frac{\partial F_{3}(\zeta(t))}{\partial y_{0}} t} \int_{0}^{t}\left(\frac{\partial^{2} F_{3}(\zeta(s))}{\partial y_{0}^{2}} \frac{\partial \Phi_{3}\left(s, \zeta_{0}\right)}{\partial y_{0}}+\frac{\partial^{2} F_{3}(\zeta(s))}{\partial y_{0} \partial y_{1}} \frac{\partial \Phi_{4}\left(s, \zeta_{0}\right)}{\partial y_{0}}\right) d s . \\
& \frac{d}{d t}\left(\frac{\partial^{2} \Phi_{3}\left(t, \zeta_{0}\right)}{\partial y_{0} \partial y_{1}}\right)=\frac{\partial F_{3}(\zeta(t))}{\partial x_{0}} \frac{\partial^{2} \Phi_{1}\left(t, \zeta_{0}\right)}{\partial y_{0} \partial y_{1}}+\frac{\partial F_{3}(\zeta(t))}{\partial x_{1}} \frac{\partial^{2} \Phi_{2}\left(t, \zeta_{0}\right)}{\partial y_{0} \partial y_{1}}+\frac{\partial F_{3}(\zeta(t))}{\partial y_{0}} \frac{\partial^{2} \Phi_{3}\left(t, \zeta_{0}\right)}{\partial y_{0} \partial y_{1}}+\frac{\partial F_{3}(\zeta(t))}{\partial y_{1}} \frac{\partial^{2} \Phi_{4}\left(t, \zeta_{0}\right)}{\partial y_{0} \partial y_{1}} \\
& +\left(\frac{\partial^{2} F_{3}(\zeta(t))}{\partial x_{0}^{2}} \frac{\partial \Phi_{1}\left(t, \zeta_{0}\right)}{\partial y_{1}}+\frac{\partial^{2} F_{3}(\zeta(t))}{\partial x_{0} \partial x_{1}} \frac{\partial \Phi_{2}\left(t, \zeta_{0}\right)}{\partial y_{1}}+\frac{\partial^{2} F_{3}(\zeta(t))}{\partial x_{0} \partial y_{0}} \frac{\partial \Phi_{3}\left(t, \zeta_{0}\right)}{\partial y_{1}}+\frac{\partial^{2} F_{3}(\zeta(t))}{\partial x_{0} \partial y_{1}} \frac{\partial \Phi_{4}\left(t, \zeta_{0}\right)}{\partial y_{1}}\right) \frac{\partial \Phi_{1}\left(t, \zeta_{0}\right)}{\partial y_{0}} \\
& +\left(\frac{\partial^{2} F_{3}(\zeta(t))}{\partial x_{0} \partial x_{1}} \frac{\partial \Phi_{1}\left(t, \zeta_{0}\right)}{\partial y_{1}}+\frac{\partial^{2} F_{3}(\zeta(t))}{\partial x_{1}^{2}} \frac{\partial \Phi_{2}\left(t, \zeta_{0}\right)}{\partial y_{1}}+\frac{\partial^{2} F_{3}(\zeta(t))}{\partial x_{1} \partial y_{0}} \frac{\partial \Phi_{3}\left(t, \zeta_{0}\right)}{\partial y_{1}}+\frac{\partial^{2} F_{3}(\zeta(t))}{\partial x_{1} \partial y_{1}} \frac{\partial \Phi_{4}\left(t, \zeta_{0}\right)}{\partial y_{1}}\right) \frac{\partial \Phi_{2}\left(t, \zeta_{0}\right)}{\partial y_{0}} \\
& +\left(\frac{\partial^{2} F_{3}(\zeta(t))}{\partial x_{0} \partial y_{0}} \frac{\partial \Phi_{1}\left(t, \zeta_{0}\right)}{\partial y_{1}}+\frac{\partial^{2} F_{3}(\zeta(t))}{\partial x_{1} \partial y_{0}} \frac{\partial \Phi_{2}\left(t, \zeta_{0}\right)}{\partial y_{1}}+\frac{\partial^{2} F_{3}(\zeta(t))}{\partial y_{0}^{2}} \frac{\partial \Phi_{3}\left(t, \zeta_{0}\right)}{\partial y_{1}}+\frac{\partial^{2} F_{3}(\zeta(t))}{\partial y_{0} \partial y_{1}} \frac{\partial \Phi_{4}\left(t, \zeta_{0}\right)}{\partial y_{1}}\right) \frac{\partial \Phi_{3}\left(t, \zeta_{0}\right)}{\partial y_{0}} \\
& +\left(\frac{\partial^{2} F_{3}(\zeta(t))}{\partial x_{0} \partial y_{1}} \frac{\partial \Phi_{1}\left(t, \zeta_{0}\right)}{\partial y_{1}}+\frac{\partial^{2} F_{3}(\zeta(t))}{\partial x_{1} \partial y_{1}} \frac{\partial \Phi_{2}\left(t, \zeta_{0}\right)}{\partial y_{1}}+\frac{\partial^{2} F_{3}(\zeta(t))}{\partial y_{0} \partial y_{1}} \frac{\partial \Phi_{3}\left(t, \zeta_{0}\right)}{\partial y_{1}}+\frac{\partial^{2} F_{3}(\zeta(t))}{\partial y_{1}^{2}} \frac{\partial \Phi_{4}\left(t, \zeta_{0}\right)}{\partial y_{1}}\right) \frac{\partial \Phi_{4}\left(t, \zeta_{0}\right)}{\partial y_{0}}
\end{aligned}
$$


with the initial condition $\frac{\partial^{2} \Phi_{3}\left(0, \zeta_{0}\right)}{\partial y_{0} \partial y_{1}}=0$, then

$$
\frac{d}{d t}\left(\frac{\partial^{2} \Phi_{3}\left(t, \zeta_{0}\right)}{\partial y_{0} \partial y_{1}}\right)=\frac{\partial F_{3}(\zeta(t))}{\partial y_{0}} \frac{\partial^{2} \Phi_{3}\left(t, \zeta_{0}\right)}{\partial y_{0} \partial y_{1}}+\frac{\partial^{2} F_{3}(\zeta(t))}{\partial y_{0} \partial y_{1}} \frac{\partial \Phi_{4}\left(t, \zeta_{0}\right)}{\partial y_{1}} \frac{\partial \Phi_{3}\left(t, \zeta_{0}\right)}{\partial y_{0}}
$$

with the initial condition $\frac{\partial^{2} \Phi_{3}\left(0, \zeta_{0}\right)}{\partial y_{0} \partial y_{1}}=0$. From (42) we have

$$
\frac{\partial^{2} \Phi_{3}\left(t, \zeta_{0}\right)}{\partial y_{0} \partial y_{1}}=e^{\frac{\partial F_{3}(\zeta(t))}{\partial y_{0}} t} \int_{0}^{t} \frac{\partial^{2} F_{3}(\zeta(s))}{\partial y_{0} \partial y_{1}} \frac{\partial \Phi_{4}\left(s, \zeta_{0}\right)}{\partial y_{1}} d s .
$$

$\frac{d}{d t}\left(\frac{\partial^{2} \Phi_{3}\left(t, \zeta_{0}\right)}{\partial y_{1}^{2}}\right)=\frac{\partial F_{3}(\zeta(t))}{\partial x_{0}} \frac{\partial^{2} \Phi_{1}\left(t, \zeta_{0}\right)}{\partial y_{1}^{2}}+\frac{\partial F_{3}(\zeta(t))}{\partial x_{1}} \frac{\partial^{2} \Phi_{2}\left(t, \zeta_{0}\right)}{\partial y_{1}^{2}}+\frac{\partial F_{3}(\zeta(t))}{\partial y_{0}} \frac{\partial^{2} \Phi_{3}\left(t, \zeta_{0}\right)}{\partial y_{1}^{2}}+\frac{\partial F_{3}(\zeta(t))}{\partial y_{1}} \frac{\partial^{2} \Phi_{4}\left(t, \zeta_{0}\right)}{\partial y_{1}^{2}}$ $+\left(\frac{\partial^{2} F_{3}(\zeta(t))}{\partial x_{0}^{2}} \frac{\partial \Phi_{1}\left(t, \zeta_{0}\right)}{\partial y_{1}}+\frac{\partial^{2} F_{3}(\zeta(t))}{\partial x_{0} \partial x_{1}} \frac{\partial \Phi_{2}\left(t, \zeta_{0}\right)}{\partial y_{1}}+\frac{\partial^{2} F_{3}(\zeta(t))}{\partial x_{0} \partial y_{0}} \frac{\partial \Phi_{3}\left(t, \zeta_{0}\right)}{\partial y_{1}}+\frac{\partial^{2} F_{3}(\zeta(t))}{\partial x_{0} \partial y_{1}} \frac{\partial \Phi_{4}\left(t, \zeta_{0}\right)}{\partial y_{1}}\right) \frac{\partial \Phi_{1}\left(t, \zeta_{0}\right)}{\partial y_{1}}$ $+\left(\frac{\partial^{2} F_{3}(\zeta(t))}{\partial x_{0} \partial x_{1}} \frac{\partial \Phi_{1}\left(t, \zeta_{0}\right)}{\partial y_{1}}+\frac{\partial^{2} F_{3}(\zeta(t))}{\partial x_{1}^{2}} \frac{\partial \Phi_{2}\left(t, \zeta_{0}\right)}{\partial y_{1}}+\frac{\partial^{2} F_{3}(\zeta(t))}{\partial x_{1} \partial y_{0}} \frac{\partial \Phi_{3}\left(t, \zeta_{0}\right)}{\partial y_{1}}+\frac{\partial^{2} F_{3}(\zeta(t))}{\partial x_{1} \partial y_{1}} \frac{\partial \Phi_{4}\left(t, \zeta_{0}\right)}{\partial y_{1}}\right) \frac{\partial \Phi_{2}\left(t, \zeta_{0}\right)}{\partial y_{1}}$ $+\left(\frac{\partial^{2} F_{3}(\zeta(t))}{\partial x_{0} \partial y_{0}} \frac{\partial \Phi_{1}\left(t, \zeta_{0}\right)}{\partial y_{1}}+\frac{\partial^{2} F_{3}(\zeta(t))}{\partial x_{1} \partial y_{0}} \frac{\partial \Phi_{2}\left(t, \zeta_{0}\right)}{\partial y_{1}}+\frac{\partial^{2} F_{3}(\zeta(t))}{\partial y_{0}^{2}} \frac{\partial \Phi_{3}\left(t, \zeta_{0}\right)}{\partial y_{1}}+\frac{\partial^{2} F_{3}(\zeta(t))}{\partial y_{0} \partial y_{1}} \frac{\partial \Phi_{4}\left(t, \zeta_{0}\right)}{\partial y_{1}}\right) \frac{\partial \Phi_{3}\left(t, \zeta_{0}\right)}{\partial y_{1}}$ $+\left(\frac{\partial^{2} F_{3}(\zeta(t))}{\partial x_{0} \partial y_{1}} \frac{\partial \Phi_{1}\left(t, \zeta_{0}\right)}{\partial y_{1}}+\frac{\partial^{2} F_{3}(\zeta(t))}{\partial x_{1} \partial y_{1}} \frac{\partial \Phi_{2}\left(t, \zeta_{0}\right)}{\partial y_{1}}+\frac{\partial^{2} F_{3}(\zeta(t))}{\partial y_{0} \partial y_{1}} \frac{\partial \Phi_{3}\left(t, \zeta_{0}\right)}{\partial y_{1}}+\frac{\partial^{2} F_{3}(\zeta(t))}{\partial y_{1}^{2}} \frac{\partial \Phi_{4}\left(t, \zeta_{0}\right)}{\partial y_{1}}\right) \frac{\partial \Phi_{4}\left(t, \zeta_{0}\right)}{\partial y_{1}}$

with the initial condition $\frac{\partial^{2} \Phi_{3}\left(0, \zeta_{0}\right)}{\partial y_{1}^{2}}=0$, then

$$
\frac{d}{d t}\left(\frac{\partial^{2} \Phi_{3}\left(t, \zeta_{0}\right)}{\partial y_{1}^{2}}\right)=\frac{\partial F_{3}(\zeta(t))}{\partial y_{0}} \frac{\partial^{2} \Phi_{3}\left(t, \zeta_{0}\right)}{\partial y_{1}^{2}}
$$

with the initial condition $\frac{\partial^{2} \Phi_{3}\left(0, \zeta_{0}\right)}{\partial y_{1}^{2}}=0$. From (43) we have

$$
\frac{\partial^{2} \Phi_{3}\left(t, \zeta_{0}\right)}{\partial y_{1}^{2}}=0
$$

\subsection{First derivatives of $Z^{*}$}

Let $\eta(\bar{\tau})=\tau_{0}+\bar{\tau}, \eta_{1}(\bar{\tau}, \sigma)=x_{0}^{*}+q_{1} \sigma+z_{1}^{*}(\bar{\tau}, \sigma), \eta_{2}(\bar{\tau}, \sigma)=\frac{a_{x}}{d_{1}} x_{0}^{*}+q_{2} \sigma+z_{2}^{*}(\bar{\tau}, \sigma)$, $\eta_{3}(\bar{\tau}, \sigma)=\sigma$ and $\eta_{4}(\bar{\tau}, \sigma)=q_{4} \sigma+z_{4}^{*}(\bar{\tau}, \sigma)$.

From (23) we have

$$
\left\{\begin{array}{l}
\frac{\partial}{\partial \bar{\tau}}\left(\eta_{1}-\Theta_{1} \circ \Phi\left(\eta, \eta_{1}, \eta_{2}, \eta_{3}, \eta_{4}\right)\right)(0,0)=0 \\
\frac{\partial}{\partial \bar{\tau}}\left(\eta_{2}-\Theta_{2} \circ \Phi\left(\eta, \eta_{1}, \eta_{2}, \eta_{3}, \eta_{4}\right)\right)(0,0)=0, \\
\frac{\partial}{\partial \bar{\tau}}\left(\eta_{4}-\Theta_{4} \circ \Phi\left(\eta, \eta_{1}, \eta_{2}, \eta_{3}, \eta_{4}\right)\right)(0,0)=0 .
\end{array}\right.
$$

Therefore

$$
\left\{\begin{aligned}
\frac{\partial z_{1}^{*}(0,0)}{\partial \bar{\tau}}-\frac{\partial \Theta_{1}\left(\Phi\left(\tau_{0}, \zeta_{0}\right)\right)}{\partial x_{0}}\left(\frac{\partial \Phi_{1}\left(\tau_{0}, \zeta_{0}\right)}{\partial \bar{\tau}}+\frac{\partial \Phi_{1}\left(\tau_{0}, \zeta_{0}\right)}{\partial x_{0}} \frac{\partial z_{1}^{*}(0,0)}{\partial \bar{\tau}}\right. & +\frac{\partial \Phi_{1}\left(\tau_{0}, \zeta_{0}\right)}{\partial x_{1}} \frac{\partial z_{2}^{*}(0,0)}{\partial \bar{\tau}} \\
\left.+\frac{\partial \Phi_{1}\left(\tau_{0}, \zeta_{0}\right)}{\partial y_{1}} \frac{\partial z_{4}^{*}(0,0)}{\partial \bar{\tau}}\right) & =0 \\
\frac{\partial z_{2}^{*}(0,0)}{\partial \bar{\tau}}-\frac{\partial \Theta_{2}\left(\Phi\left(\tau_{0}, \zeta_{0}\right)\right)}{\partial x_{1}}\left(\frac{\partial \Phi_{2}\left(\tau_{0}, \zeta_{0}\right)}{\partial \bar{\tau}}+\frac{\partial \Phi_{2}\left(\tau_{0}, \zeta_{0}\right)}{\partial x_{0}} \frac{\partial z_{1}^{*}(0,0)}{\partial \bar{\tau}}\right. & +\frac{\partial \Phi_{2}\left(\tau_{0}, \zeta_{0}\right)}{\partial x_{1}} \frac{\partial z_{2}^{*}(0,0)}{\partial \bar{\tau}} \\
\left.+\frac{\partial \Phi_{2}\left(\tau_{0}, \zeta_{0}\right)}{\partial y_{1}} \frac{\partial z_{4}^{*}(0,0)}{\partial \bar{\tau}}\right) & =0 \\
+\frac{\partial \Phi_{4}\left(\tau_{0}, \zeta_{0}\right)}{\partial x_{0}} \frac{\partial z_{1}^{*}(0,0)}{\partial \bar{\tau}} & +\frac{\partial \Phi_{4}\left(\tau_{0}, \zeta_{0}\right)}{\partial x_{1}} \frac{\partial z_{2}^{*}(0,0)}{\partial \bar{\tau}} \\
\left.+\frac{\partial \Phi_{4}\left(\tau_{0}, \zeta_{0}\right)}{\partial y_{1}} \frac{\partial z_{4}^{*}(0,0)}{\partial \bar{\tau}}\right) & =0 .
\end{aligned}\right.
$$


Since $\frac{\partial \Phi_{1}\left(\tau_{0}, \zeta_{0}\right)}{\partial \bar{\tau}}=\frac{\partial \Phi_{2}\left(\tau_{0}, \zeta_{0}\right)}{\partial \bar{\tau}}=\frac{\partial \Phi_{4}\left(\tau_{0}, \zeta_{0}\right)}{\partial \bar{\tau}}=0$, we obtain

$$
\left\{\begin{array}{l}
a_{0} \frac{\partial z_{1}^{*}(0,0)}{\partial \bar{\tau}}+b_{0} \frac{\partial z_{2}^{*}(0,0)}{\partial \bar{\tau}}+d_{0} \frac{\partial z_{4}^{*}(0,0)}{\partial \bar{\tau}}=0 \\
e_{0} \frac{\partial z_{1}^{*}(0,0)}{\partial \bar{\tau}}+f_{0} \frac{\partial z_{2}^{*}(0,0)}{\partial \bar{\tau}}=0 \\
i_{0} \frac{\partial z_{4}^{*}(0,0)}{\partial \bar{\tau}}=0
\end{array}\right.
$$

That is

$$
\left\{\begin{aligned}
\frac{\partial z_{1}^{*}(0,0)}{\partial \bar{\tau}} & =0 \\
\frac{\partial z_{2}^{*}(0,0)}{\partial \bar{\tau}} & =0 \\
\frac{\partial z_{4}^{*}(0,0)}{\partial \bar{\tau}} & =0
\end{aligned}\right.
$$

In the same way as above, we obtain

$$
\left\{\begin{array}{l}
\frac{\partial}{\partial g}\left(\eta_{1}-\Theta_{1} \circ \Phi\left(\eta, \eta_{1}, \eta_{2}, \eta_{3}, \eta_{4}\right)\right)(0,0)=0 \\
\frac{\partial}{\partial \sigma}\left(\eta_{2}-\Theta_{2} \circ \Phi\left(\eta, \eta_{1}, \eta_{2}, \eta_{3}, \eta_{4}\right)\right)(0,0)=0 \\
\frac{\partial}{\partial \sigma}\left(\eta_{4}-\Theta_{4} \circ \Phi\left(\eta, \eta_{1}, \eta_{2}, \eta_{3}, \eta_{4}\right)\right)(0,0)=0 .
\end{array}\right.
$$

Therefore

$$
\left\{\begin{aligned}
\frac{\partial z_{1}^{*}(0,0)}{\partial \sigma}-\frac{\partial \Theta_{1}\left(\Phi\left(\tau_{0}, \zeta_{0}\right)\right)}{\partial x_{0}}\left(\frac { \partial \Phi _ { 1 } ( \tau _ { 0 } , \zeta _ { 0 } ) } { \partial x _ { 0 } } \left(q_{1}+\right.\right. & \left.\frac{\partial z_{1}^{*}(0,0)}{\partial \sigma}\right)+\frac{\partial \Phi_{1}\left(\tau_{0}, \zeta_{0}\right)}{\partial x_{1}}\left(q_{2}+\frac{\partial z_{2}^{*}(0,0)}{\partial \sigma}\right) \\
& \left.+\frac{\partial \Phi_{1}\left(\tau_{0}, \zeta_{0}\right)}{\partial y_{0}}+\frac{\partial \Phi_{1}\left(\tau_{0}, \zeta_{0}\right)}{\partial y_{1}}\left(q_{1}+\frac{\partial z_{4}^{*}(0,0)}{\partial \sigma}\right)\right)=0 \\
\frac{\partial z_{2}^{*}(0,0)}{\partial \sigma}-\frac{\partial \Theta_{2}\left(\Phi\left(\tau_{0}, \zeta_{0}\right)\right)}{\partial x_{0}}\left(\frac { \partial \Phi _ { 2 } ( \tau _ { 0 } , \zeta _ { 0 } ) } { \partial x _ { 0 } } \left(q_{1}+\right.\right. & \left.\frac{\partial z_{1}^{*}(0,0)}{\partial \sigma}\right)+\frac{\partial \Phi_{2}\left(\tau_{0}, \zeta_{0}\right)}{\partial x_{1}}\left(q_{2}+\frac{\partial z_{2}^{*}(0,0)}{\partial \sigma}\right) \\
& \left.+\frac{\partial \Phi_{2}\left(\tau_{0}, \zeta_{0}\right)}{\partial y_{0}}+\frac{\partial \Phi_{2}\left(\tau_{0}, \zeta_{0}\right)}{\partial y_{1}}\left(q_{1}+\frac{\partial z_{4}^{*}(0,0)}{\partial \sigma}\right)\right)=0, \\
\frac{\partial z_{4}^{*}(0,0)}{\partial \sigma}-\frac{\partial \Theta_{4}\left(\Phi\left(\tau_{0}, \zeta_{0}\right)\right)}{\partial x_{0}}\left(\frac { \partial \Phi _ { 4 } ( \tau _ { 0 } , \zeta _ { 0 } ) } { \partial x _ { 0 } } \left(q_{1}+\right.\right. & \left.\frac{\partial z_{1}^{*}(0,0)}{\partial \sigma}\right)+\frac{\partial \Phi_{4}\left(\tau_{0}, \zeta_{0}\right)}{\partial x_{1}}\left(q_{2}+\frac{\partial z_{2}^{*}(0,0)}{\partial \sigma}\right) \\
& \left.+\frac{\partial \Phi_{4}\left(\tau_{0}, \zeta_{0}\right)}{\partial y_{0}}+\frac{\partial \Phi_{4}\left(\tau_{0}, \zeta_{0}\right)}{\partial y_{1}}\left(q_{1}+\frac{\partial z_{4}^{*}(0,0)}{\partial \sigma}\right)\right)=0 .
\end{aligned}\right.
$$

We obtain

$$
\left\{\begin{array}{l}
a_{0} \frac{\partial z_{1}^{*}(0,0)}{\partial \sigma}+b_{0} \frac{\partial z_{2}^{*}(0,0)}{\partial \sigma}+c_{0}+d_{0} \frac{\partial z_{4}^{*}(0,0)}{\partial \sigma}=0 \\
e_{0} \frac{\partial z_{1}^{*}(0,0)}{\partial \sigma}+f_{0} \frac{\partial z_{2}^{*}(0,0)}{\partial \sigma}=0 \\
i_{0} \frac{\partial z_{4}^{*}(0,0)}{\partial \sigma}=0
\end{array}\right.
$$

That is

$$
\left\{\begin{aligned}
\frac{\partial z_{1}^{*}(0,0)}{\partial \sigma} & =-\frac{f_{0} c_{0}}{a_{0} f_{0}-b_{0} e_{0}} \\
\frac{\partial z_{2}^{*}(0,0)}{\partial \sigma} & =\frac{e_{0} c_{0}}{a_{0} f_{0}-b_{0} e_{0}} \\
\frac{\partial z_{4}^{*}(0,0)}{\partial \sigma} & =0
\end{aligned}\right.
$$

\subsection{First derivatives of $\omega$}

We have

$$
\begin{aligned}
\frac{\partial \omega}{\partial \bar{\tau}}= & \frac{\partial}{\partial \bar{\tau}}\left(\eta_{3}-\Theta_{3} \circ \Phi\left(\eta, \eta_{1}, \eta_{2}, \eta_{3}, \eta_{4}\right)\right) \\
= & -\frac{\partial \Theta_{3}}{\partial y_{0}}\left(\frac{\partial \Phi_{3}\left(\eta, \eta_{1}, \eta_{2}, \eta_{3}, \eta_{4}\right)}{\partial \bar{\tau}}+\frac{\partial \Phi_{3}\left(\eta, \eta_{1}, \eta_{2}, \eta_{3}, \eta_{4}\right)}{\partial x_{0}} \frac{\partial z_{1}^{*}}{\partial \bar{\tau}}+\frac{\partial \Phi_{3}\left(\eta, \eta_{1}, \eta_{2}, \eta_{3}, \eta_{4}\right)}{\partial x_{1}} \frac{\partial z_{2}^{*}}{\partial \bar{\tau}}\right. \\
& \left.+\frac{\partial \Phi_{3}\left(\eta, \eta_{1}, \eta_{2}, \eta_{3}, \eta_{4}\right)}{\partial y_{1}} \frac{\partial z_{4}^{*}}{\partial \bar{\tau}}\right) .
\end{aligned}
$$

At $(\bar{\tau}, \sigma)=(0,0)$ we have $\frac{\partial \Phi_{3}\left(\tau_{0}, \zeta_{0}\right)}{\partial \bar{\tau}}=0$, then we obtain

$$
\frac{\partial \omega}{\partial \bar{\tau}}(0,0)=-\frac{\partial \Theta_{3}}{\partial y_{0}}\left(\frac{\partial \Phi_{3}\left(\tau_{0}, \zeta_{0}\right)}{\partial x_{0}} \frac{\partial z_{1}^{*}(0,0)}{\partial \bar{\tau}}+\frac{\partial \Phi_{3}\left(\tau_{0}, \zeta_{0}\right)}{\partial x_{1}} \frac{\partial z_{2}^{*}(0,0)}{\partial \bar{\tau}}+\frac{\partial \Phi_{3}\left(\tau_{0}, \zeta_{0}\right)}{\partial y_{1}} \frac{\partial z_{4}^{*}(0,0)}{\partial \bar{\tau}}\right)=0 .
$$




$$
\begin{aligned}
\frac{\partial \omega}{\partial \sigma}= & \frac{\partial}{\partial \sigma}\left(\eta_{3}-\Theta_{3} \circ \Phi\left(\eta, \eta_{1}, \eta_{2}, \eta_{3}, \eta_{4}\right)\right) \\
= & 1-\frac{\partial \Theta_{3}}{\partial y_{0}}\left(\frac{\partial \Phi_{3}\left(\eta, \eta_{1}, \eta_{2}, \eta_{3}, \eta_{4}\right)}{\partial x_{0}}\left(q_{1}+\frac{\partial z_{1}^{*}}{\partial \sigma}\right)+\frac{\partial \Phi_{3}\left(\eta, \eta_{1}, \eta_{2}, \eta_{3}, \eta_{4}\right)}{\partial x_{1}}\left(q_{2}+\frac{\partial z_{2}^{*}}{\partial \sigma}\right)\right. \\
& \left.\quad+\frac{\partial \Phi_{3}\left(\eta, \eta_{1}, \eta_{2}, \eta_{3}, \eta_{4}\right)}{\partial y_{0}}+\frac{\partial \Phi_{3}\left(\eta, \eta_{1}, \eta_{2}, \eta_{3}, \eta_{4}\right)}{\partial y_{1}}\left(q_{4}+\frac{\partial z_{4}^{*}}{\partial \sigma}\right)\right) .
\end{aligned}
$$

At $(\bar{\tau}, \sigma)=(0,0)$ we obtain

$$
\begin{aligned}
\frac{\partial \omega}{\partial \sigma}(0,0)= & 1-\frac{\partial \Theta_{3}}{\partial y_{0}}\left(\frac{\partial \Phi_{3}\left(\tau_{0}, \zeta_{0}\right)}{\partial 0_{0}}\left(q_{1}+\frac{\partial z_{1}^{*}(0,0)}{\partial \sigma}\right)+\frac{\partial \Phi_{3}\left(\tau_{0}, \zeta_{0}\right)}{\partial x_{1}}\left(q_{2}+\frac{\partial z_{2}^{*}(0,0)}{\partial \sigma}\right)\right. \\
& \left.+\frac{\partial \Phi_{3}\left(\tau_{0}, \zeta_{0}\right)}{\partial y_{0}}+\frac{\partial \Phi_{3}\left(\tau_{0}, \zeta_{0}\right)}{\partial y_{1}}\left(q_{4}+\frac{\partial z_{4}^{*}(0,0)}{\partial \sigma}\right)\right) \\
= & 1-\frac{\partial \Theta_{3}}{\partial y_{0}} \frac{\partial \Phi_{3}\left(\tau_{0}, \zeta_{0}\right)}{\partial y_{0}} \\
= & g_{0}=0
\end{aligned}
$$

Therefore $D_{(\bar{\tau}, \sigma)} \omega(0,0)=(0,0)$.

\subsection{Second derivatives of $\omega$}

Let $\mathcal{A}=\frac{\partial^{2} \omega(0,0)}{\partial \bar{\tau}^{2}}, \mathcal{B}=\frac{\partial^{2} \omega(0,0)}{\partial \bar{\tau} \partial \sigma}$ and $\mathcal{C}=\frac{\partial^{2} \omega(0,0)}{\partial \sigma^{2}}$.

\subsubsection{Calculation of $\mathcal{A}$.}

We have $\frac{\partial^{2} \omega}{\partial \bar{\tau}^{2}}=\frac{\partial^{2}}{\partial \bar{\tau}^{2}}\left(\eta_{3}-\Theta_{3} \circ \Phi\left(\eta, \eta_{1}, \eta_{2}, \eta_{3}, \eta_{4}\right)\right)$, then

$$
\begin{aligned}
& \frac{\partial^{2} \omega}{\partial \bar{\tau}^{2}}=-\frac{\partial \Theta_{3}}{\partial y_{0}}\left\{\frac{\partial^{2} \Phi_{3}\left(\eta, \eta_{1}, \eta_{2}, \eta_{3}, \eta_{4}\right)}{\partial \bar{\tau}^{2}}+2 \frac{\partial^{2} \Phi_{3}\left(\eta, \eta_{1}, \eta_{2}, \eta_{3}, \eta_{4}\right)}{\partial \bar{\tau} \partial x_{0}} \frac{\partial z_{1}^{*}}{\partial \bar{\tau}}+2 \frac{\partial^{2} \Phi_{3}\left(\eta, \eta_{1}, \eta_{2}, \eta_{3}, \eta_{4}\right)}{\partial \bar{\tau} \partial x_{1}} \frac{\partial z_{2}^{*}}{\partial \bar{\tau}}\right. \\
& +2 \frac{\partial^{2} \Phi_{3}\left(\eta, \eta_{1}, \eta_{2}, \eta_{3}, \eta_{4}\right)}{\partial \bar{\tau} \partial y_{1}} \frac{\partial z_{4}^{*}}{\partial \bar{\tau}}+\frac{\partial^{2} \Phi_{3}\left(\eta, \eta_{1}, \eta_{2}, \eta_{3}, \eta_{4}\right)}{\partial x_{0}^{2}}\left(\frac{\partial z_{1}^{*}}{\partial \bar{\tau}}\right)^{2}+2 \frac{\partial^{2} \Phi_{3}\left(\eta, \eta_{1}, \eta_{2}, \eta_{3}, \eta_{4}\right)}{\partial x_{0} \partial x_{1}} \frac{\partial z_{1}^{*}}{\partial \bar{\tau}} \frac{\partial z_{2}^{*}}{\partial \bar{\tau}} \\
& +2 \frac{\partial^{2} \Phi_{3}\left(\eta, \eta_{1}, \eta_{2}, \eta_{3}, \eta_{4}\right)}{\partial x_{0} \partial y_{1}} \frac{\partial z_{1}^{*}}{\partial \bar{\tau}} \frac{\partial z_{4}^{*}}{\partial \bar{\tau}}+\frac{\partial \Phi_{3}\left(\eta, \eta_{1}, \eta_{2}, \eta_{3}, \eta_{4}\right)}{\partial x_{0}} \frac{\partial^{2} z_{1}^{*}}{\partial \bar{\tau}^{2}}+\frac{\partial^{2} \Phi_{3}\left(\eta, \eta_{1}, \eta_{2}, \eta_{3}, \eta_{4}\right)}{\partial x_{1}^{2}}\left(\frac{\partial z_{2}^{*}}{\partial \bar{\tau}}\right)^{2} \\
& +2 \frac{\partial^{2} \Phi_{3}\left(\eta, \eta_{1}, \eta_{2}, \eta_{3}, \eta_{4}\right)}{\partial x_{1} \partial y_{1}} \frac{\partial z_{2}^{*}}{\partial \bar{\tau}} \frac{\partial z_{4}^{*}}{\partial \bar{\tau}}+\frac{\partial \Phi_{3}\left(\eta, \eta_{1}, \eta_{2}, \eta_{3}, \eta_{4}\right)}{\partial x_{1}} \frac{\partial^{2} z_{2}^{*}}{\partial \bar{\tau}^{2}}+\frac{\partial^{2} \Phi_{3}\left(\eta, \eta_{1}, \eta_{2}, \eta_{3}, \eta_{4}\right)}{\partial y_{1}^{2}}\left(\frac{\partial z_{4}^{*}}{\partial \bar{\tau}}\right)^{2} \\
& \left.+\frac{\partial \Phi_{3}\left(\eta, \eta_{1}, \eta_{2}, \eta_{3}, \eta_{4}\right)}{\partial y_{1}} \frac{\partial^{2} z_{4}^{*}}{\partial \bar{\tau}^{2}}\right\} .
\end{aligned}
$$

At $(\bar{\tau}, \sigma)=(0,0)$ we have $\frac{\partial^{2} \Phi_{3}\left(\tau_{0}, \zeta_{0}\right)}{\partial \bar{\tau}^{2}}=0$. Then

$$
\mathcal{A}=0 \text {. }
$$

\subsubsection{Calculation of $\mathcal{C}$.}

We have $\frac{\partial^{2} \omega}{\partial \sigma^{2}}=\frac{\partial^{2}}{\partial \sigma^{2}}\left(\eta_{3}-\Theta_{3} \circ \Phi\left(\eta, \eta_{1}, \eta_{2}, \eta_{3}, \eta_{4}\right)\right)$, then

$$
\begin{aligned}
\frac{\partial^{2} \omega}{\partial \sigma^{2}}= & -\frac{\partial \Theta_{3}}{\partial y_{0}}\left(\frac{\partial^{2} \Phi_{3}\left(\eta, \eta_{1}, \eta_{2}, \eta_{3}, \eta_{4}\right)}{\partial x_{0}^{2}}\left(q_{1}+\frac{\partial z_{1}^{*}}{\partial \sigma}\right)^{2}+2 \frac{\partial^{2} \Phi_{3}\left(\eta, \eta_{1}, \eta_{2}, \eta_{3}, \eta_{4}\right)}{\partial x_{0} \partial x_{1}}\left(q_{1}+\frac{\partial z_{1}^{*}}{\partial \sigma}\right)\left(q_{2}+\frac{\partial z_{2}^{*}}{\partial \sigma}\right)\right. \\
& +2 \frac{\partial^{2} \Phi_{3}\left(\eta, \eta_{1}, \eta_{2}, \eta_{3}, \eta_{4}\right)}{\partial x_{0} \partial y_{1}}\left(q_{1}+\frac{\partial z_{1}^{*}}{\partial \sigma}\right)\left(q_{4}+\frac{\partial z_{4}^{*}}{\partial \sigma}\right)+2 \frac{\partial^{2} \Phi_{3}\left(\eta, \eta_{1}, \eta_{2}, \eta_{3}, \eta_{4}\right)}{\partial x_{0} \partial y_{0}}\left(q_{1}+\frac{\partial z_{1}^{*}}{\partial \sigma}\right) \\
& +\frac{\partial \Phi_{3}\left(\eta, \eta_{1}, \eta_{2}, \eta_{3}, \eta_{4}\right)}{\partial x_{0}}\left(\frac{\partial^{2} z_{1}^{*}}{\partial \sigma^{2}}\right)+\frac{\partial^{2} \Phi_{3}\left(\eta, \eta_{1}, \eta_{2}, \eta_{3}, \eta_{4}\right)}{\partial x_{1}^{2}}\left(q_{2}+\frac{\partial z_{2}^{*}}{\partial \sigma}\right)^{2} \\
& +2 \frac{\partial^{2} \Phi_{3}\left(\eta, \eta_{1}, \eta_{2}, \eta_{3}, \eta_{4}\right)}{\partial x_{1} \partial y_{1}}\left(q_{2}+\frac{\partial z_{2}^{*}}{\partial \sigma}\right)\left(q_{4}+\frac{\partial z_{4}^{*}}{\partial \sigma}\right)+2 \frac{\partial^{2} \Phi_{3}\left(\eta, \eta_{1}, \eta_{2}, \eta_{3}, \eta_{4}\right)}{\partial y_{0} \partial x_{1}}\left(q_{2}+\frac{\partial z_{2}^{*}}{\partial \sigma}\right) \\
& +\frac{\partial \Phi_{3}\left(\eta, \eta_{1}, \eta_{2}, \eta_{3}, \eta_{4}\right)}{\partial x_{1}}\left(\frac{\partial^{2} z_{2}^{*}}{\partial \sigma^{2}}\right)+\frac{\partial^{2} \Phi_{3}\left(\eta, \eta_{1}, \eta_{2}, \eta_{3}, \eta_{4}\right)}{\partial y_{1}^{2}}\left(\frac{\partial z_{4}^{*}}{\partial \sigma}\right)^{2}+2 \frac{\partial^{2} \Phi_{3}\left(\eta, \eta_{1}, \eta_{2}, \eta_{3}, \eta_{4}\right)}{\partial y_{0} \partial y_{1}}\left(q_{4}+\frac{\partial z_{4}^{*}}{\partial \sigma}\right) \\
& \left.+\frac{\partial \Phi_{3}\left(\eta, \eta_{1}, \eta_{2}, \eta_{3}, \eta_{4}\right)}{\partial y_{1}}\left(\frac{\partial^{2} z_{4}^{*}}{\partial \sigma^{2}}\right)+\frac{\partial^{2} \Phi_{3}\left(\eta, \eta_{1}, \eta_{2}, \eta_{3}, \eta_{4}\right)}{\partial y_{0}^{2}}\right\} .
\end{aligned}
$$


At $(\bar{\tau}, \sigma)=(0,0)$ we obtain

$$
\begin{aligned}
\mathcal{C}= & -\frac{\partial \Theta_{3}}{\partial y_{0}}\left\{2 \frac{\partial^{2} \Phi_{3}\left(\tau_{0}, \zeta_{0}\right)}{\partial x_{0} \partial y_{0}}\left(q_{1}+\frac{\partial z_{1}^{*}(0,0)}{\partial \sigma}\right)+2 \frac{\partial^{2} \Phi_{3}\left(\tau_{0}, \zeta_{0}\right)}{\partial x_{1} \partial y_{0}}\left(q_{2}+\frac{\partial z_{2}^{*}(0,0)}{\partial \sigma}\right)\right. \\
& \left.+2 \frac{\partial^{2} \Phi_{3}\left(\tau_{0}, \zeta_{0}\right)}{\partial y_{0} \partial y_{1}} q_{4}+\frac{\partial^{2} \Phi_{3}\left(\tau_{0}, \zeta_{0}\right)}{\partial y_{0}^{2}}\right\} .
\end{aligned}
$$

\subsubsection{Calculation of $\mathcal{B}$.}

We have $\frac{\partial^{2} \omega}{\partial \bar{\tau} \partial \sigma}=\frac{\partial}{\partial \bar{\tau}}\left(\frac{\partial}{\partial \sigma}\left(\eta_{3}-\Theta_{3} \circ \Phi\left(\eta, \eta_{1}, \eta_{2}, \eta_{3}, \eta_{4}\right)\right)\right)$, then

$$
\begin{aligned}
\frac{\partial^{2} \omega}{\partial \bar{\tau} \partial \sigma}= & -\frac{\partial \Theta_{3}}{\partial y_{0}}\left\{\frac{\partial^{2} \Phi_{3}\left(\eta, \eta_{1}, \eta_{2}, \eta_{3}, \eta_{4}\right)}{\partial \bar{\tau} \partial x_{0}}\left(q_{1}+\frac{\partial z_{1}^{*}}{\partial \sigma}\right)+\frac{\partial^{2} \Phi_{3}\left(\eta, \eta_{1}, \eta_{2}, \eta_{3}, \eta_{4}\right)}{\partial \bar{\tau} \partial x_{1}}\left(q_{2}+\frac{\partial z_{2}^{*}}{\partial \sigma}\right)\right. \\
& +\frac{\partial^{2} \Phi_{3}\left(\eta, \eta_{1}, \eta_{2}, \eta_{3}, \eta_{4}\right)}{\partial \bar{\tau} \partial y_{0}}+\frac{\partial^{2} \Phi_{3}\left(\eta, \eta_{1}, \eta_{2}, \eta_{3}, \eta_{4}\right)}{\partial \bar{\tau} \partial y_{1}}\left(q_{4}+\frac{\partial z_{4}^{*}}{\partial \sigma}\right) \\
& +\frac{\partial^{2} \Phi_{3}\left(\eta, \eta_{1}, \eta_{2}, \eta_{3}, \eta_{4}\right)}{\partial x_{0}^{2}} \frac{\partial z_{1}^{*}}{\partial \bar{\tau}}\left(q_{1}+\frac{\partial z_{1}^{*}}{\partial \sigma}\right)+\frac{\partial^{2} \Phi_{3}\left(\eta, \eta_{1}, \eta_{2}, \eta_{3}, \eta_{4}\right)}{\partial x_{0} \partial x_{1}} \frac{\partial z_{1}^{*}}{\partial \bar{\tau}}\left(q_{2}+\frac{\partial z_{2}^{*}}{\partial \sigma}\right) \\
& +\frac{\partial^{2} \Phi_{3}\left(\eta, \eta_{1}, \eta_{2}, \eta_{3}, \eta_{4}\right)}{\partial y_{0} \partial x_{0}} \frac{\partial z_{1}^{*}}{\partial \bar{\tau}}+\frac{\partial^{2} \Phi_{3}\left(\eta, \eta_{1}, \eta_{2}, \eta_{3}, \eta_{4}\right)}{\partial y_{1} \partial x_{0}} \frac{\partial z_{1}^{*}}{\partial \bar{\tau}}\left(q_{4}+\frac{\partial z_{4}^{*}}{\partial \sigma}\right) \\
& +\frac{\partial \Phi_{3}\left(\eta, \eta_{1}, \eta_{2}, \eta_{3}, \eta_{4}\right)}{\partial x_{0}} \frac{\partial^{2} z_{1}^{*}}{\partial \bar{\tau} \partial \sigma}+\frac{\partial^{2} \Phi_{3}\left(\eta, \eta_{1}, \eta_{2}, \eta_{3}, \eta_{4}\right)}{\partial x_{0} \partial z_{1}} \frac{\partial z_{1}^{*}}{\partial \bar{\tau}}\left(q_{1}+\frac{\partial z_{1}^{*}}{\partial \sigma}\right) \\
& +\frac{\partial^{2} \Phi_{3}\left(\eta, \eta_{1}, \eta_{2}, \eta_{3}, \eta_{4}\right)}{\partial x_{1}^{2}} \frac{\partial z_{2}^{*}}{\partial \bar{\tau}}\left(q_{2}+\frac{\partial z_{2}^{*}}{\partial \sigma}\right)+\frac{\partial^{2} \Phi_{3}\left(\eta, \eta_{1}, \eta_{2}, \eta_{3}, \eta_{4}\right)}{\partial x_{1} \partial y_{0}} \frac{\partial z_{2}^{*}}{\partial \bar{\tau}} \\
& +\frac{\partial^{2} \Phi_{3}\left(\eta, \eta_{1}, \eta_{2}, \eta_{3}, \eta_{4}\right)}{\partial x_{1} \partial y_{1}} \frac{\partial z_{2}^{*}}{\partial \bar{\tau}}\left(q_{4}+\frac{\partial z_{4}^{*}}{\partial \sigma}\right)+\frac{\partial \Phi_{3}\left(\eta, \eta_{1}, \eta_{2}, \eta_{3}, \eta_{4}\right)}{\partial x_{1}} \frac{\partial^{2} z_{2}^{*}}{\partial \bar{\tau} \partial \sigma} \\
& +\frac{\partial^{2} \Phi_{3}\left(\eta, \eta_{1}, \eta_{2}, \eta_{3}, \eta_{4}\right)}{\partial y_{1} \partial x_{0}} \frac{\partial z_{4}^{*}}{\partial \bar{\tau}}\left(q_{1}+\frac{\partial z_{1}^{*}}{\partial \sigma}\right)+\frac{\partial^{2} \Phi_{3}\left(\eta, \eta_{1}, \eta_{2}, \eta_{3}, \eta_{4}\right)}{\partial y_{1} \partial x_{1}} \frac{\partial z_{4}^{*}}{\partial \bar{\tau}}\left(q_{2}+\frac{\partial z_{2}^{*}}{\partial \sigma}\right) \\
& +\frac{\partial^{2} \Phi_{3}\left(\eta, \eta_{1}, \eta_{2}, \eta_{3}, \eta_{4}\right)}{\partial y_{1}^{2}} \frac{\partial z_{1}^{*}}{\partial \bar{\tau}}\left(q_{4}+\frac{\partial z_{4}^{*}}{\partial \sigma}\right)+\frac{\partial^{2} \Phi_{3}\left(\eta, \eta_{1}, \eta_{2}, \eta_{3}, \eta_{4}\right)}{\partial y_{0} \partial y_{1}} \frac{\partial z_{4}^{*}}{\partial \bar{\tau}} \\
& \left.+\frac{\partial \Phi_{3}\left(\eta, \eta_{1}, \eta_{2}, \eta_{3}, \eta_{4}\right)}{\partial y_{1}} \frac{\partial^{2} z_{4}^{*}}{\partial \bar{\tau} \partial \sigma}\right\}
\end{aligned}
$$

At $(\bar{\tau}, \sigma)=(0,0)$ we have $\frac{\partial^{2} \Phi_{3}\left(\tau_{0}, \zeta_{0}\right)}{\partial \bar{\tau} \partial x_{0}}=\frac{\partial^{2} \Phi_{3}\left(\tau_{0}, \zeta_{0}\right)}{\partial \bar{\tau} \partial x_{1}}=\frac{\partial^{2} \Phi_{3}\left(\tau_{0}, \zeta_{0}\right)}{\partial \bar{\tau} \partial y_{1}}=0$, then we obtain

$$
\mathcal{B}=-\frac{\partial \Theta_{3}}{\partial y_{0}} \frac{\partial^{2} \Phi_{3}\left(\tau_{0}, \zeta_{0}\right)}{\partial \bar{\tau} \partial y_{0}} .
$$

\section{5. references}

[1] D. D. BAinov And P. S. Simeonov, « Oscillation Theory of Impulsive Differential Equations », International Publications, Orlando, Fla, USA, 1998.

[2] D. D. BAinov AND P. S. Simeonov, «Impulsive Differential Equations : Asymptotic Properties of the Solutions », vol. of Series on Advances in Mathematics for Applied Sciences, World Scientific, Singapore, 1995.

[3] D. BAinov AND V. CovacheV, «Impulsive Differential Equations with a Small Parameter », vol. 24 of Series on Advances in Mathematics for Applied Sciences, World Scientific, Singapore, 1994.

[4] D. D. BAINOV AND P. S. SIMEONOV, «Impulsive differential equations : periodic solutions and applications », Longman Scientific and Technical, Essex, England, 1993.

[5] D. D. Bainov, V. Lakshmikantham And P. S. Simeonov, « Theory of Impulsive Differential Equations », vol. 6 of Series in Modern Applied Mathematics, World Scientific, Singapore, 1989.

[6] D. D. BAInOv AND P.S. Simenov, « Systems with Impulse Effect Stability Theory and Applications », Ellis Horwood Limited, Chichester, 1989. 
[7] A. Boudermine, M. Helal and A. Lakmeche, « Bifurcation of non trivial periodic solutions for pulsed chemotherapy model », Journal of Mathematical Sciences and Applications, E-Notes, vol. 2, n ${ }^{\circ} 2,2014,22-44$.

[8] A. Dishliev AND D. D. BAINov, « Dependence upon initial conditions and parameters of solutions of impulsive differential equations with variable structure », International Journal of Theoretical Physics, vol. 29, 1990, 655-676.

[9] M. Helal, M. Adimy, A Lakmeche and L. P. Menjouet, « Analysis of mathematical model of leukemia », ITM Web of Conferences, vol. 4, $\mathrm{n}^{\circ}$ 01005, 2015, 1-14.

[10] G. Iooss, «Bifurcation of maps and applications », Study of mathematics, North Holland, 1979.

[11] A. LAKMECHE AND O. ARINO, «Bifurcation of nontrivial periodic solutions of impulsive differential equations arising in chemotherapeutic treatment », Dynamics Cont. Discr. Impl. Syst., vol. 7, 2000, 265-287.

[12] A. LAKMECHE AND O. ARINO, « Nonlinear mathematical model of pulsed-therapy of hetergenous tumor », Nonlinear Anal. Real World Appl., vol. 2, 2001, 455-465.

[13] Ah. Lakmeche, M. Helal AND A. LAKMECHE, «Pulsed chemotherapy treatment», Electronic Journal of Mathematical Analysis and Applications, vol. 2, $\mathrm{n}^{\circ}$ 1, 2014, 127-148.

[14] P. KIM, P. LEE AND D. LEVY, « Dynamics and potential impact of the immune response to chronic myelogenous leukemia », PLoS Comput. Biol., vol. 4, nº 6, 2008, e1000095.

[15] N. L. Komarova AND D. WodArz, « Drug resistance in cancer : Principles of emergence and prevention », Proc Natl Acad Sci USA, vol. 102, n 27,2005, 9714-9719.

[16] F. Michor, T.P. Hughes, Y. Iwasa, S. Branford, N.P Shah, C.L. SaWyers ANd M. A. NOWAK, « Dynamic of chronic myeloid leukemia », Naure vol. 435, 2005, 1267-1270.

[17] I. Roeder, M. Horn, I. Glauche, A. Hochhaus, M. C. Mueller And M. Loeffler, « Dynamic modeling of IMATINIB-treated chronic myeloid leukemia, functional insights and clinical implications », Nat Med., vol. 12, $\mathrm{n}^{\circ}$ 10, 2006, 1181-1184. 